\title{
The Limits of Hamiltonian Structures in Three Dimensional Elasticity, Shells, and Rods
}

\author{
Zhong Ge $^{*} \quad$ Hans Peter Kruse ${ }^{\dagger} \quad$ Jerrold E. Marsden ${ }^{\ddagger}$ \\ March, 1994; this version: July 20, 1998 \\ J. Nonlinear Sci. 6, (1996), 19-57
}

\begin{abstract}
This paper uses Hamiltonian structures to study the problem of the limit of three dimensional elastic models to shell and rod models. In the case of shells, we show that the Hamiltonian structure for a three dimensional elastic body converges, in a sense made precise, to that for a shell model described by a one-director Cosserat surface as the thickness goes to zero. We study limiting procedures that give rise to unconstrained as well as constrained Cosserat director models. The case of a rod is also considered and similar convergence results are established, with the limiting model being a geometrically exact director rod model (in the framework developed by Antman, Simo and coworkers). The resulting model may have constraints or not, depending on the nature of the constitutive relations and their behavior under the limiting procedure.

The closeness of Hamiltonian structures is measured by the closeness of Poisson brackets on certain classes of functions, as well as of the Hamiltonians. This provides one way of justifying the dynamic one-director model for shells. Another way of stating the convergence result is that there is an almost-Poisson embedding from the phase space of the shell to the phase space of the 3d elastic body, which implies that, in the sense of Hamiltonian structures, the dynamics of the elastic body is close to that of the shell. The constitutive equations of the $3 \mathrm{~d}$-model and their behavior as the thickness tends to zero dictates whether the limiting $2 \mathrm{~d}$-model is a constrained or an unconstrained director model.

We apply our theory in the specific case of a 3d Saint Venant-Kirchhoff material and derive the corresponding limiting shell and rod theories. The limiting shell model is an interesting Kirchhoff like shell model in which the stored energy function is explicitly derived in terms of the shell curvature. For rods, one gets (with an additional inextensibility constraint) a onedirector Kirchhoff elastic rod model, which reduces to the well-known Euler elastica if one adds an additional single constraint that the director lines up with the Frenet frame.
\end{abstract}

\footnotetext{
${ }^{*}$ The Fields Institute for Research in Mathematical Sciences, 185 Columbia St. West, Waterloo, Ontario N2L 5Z5, email: a2504gez@cdf.toronto.edu

†Zentrum Mathematik, TU München, Arcisstrasse 21, D-80290 München, Germany, email: kruse@mathematik.tumuenchen.de

${ }^{\ddagger}$ Control and Dynamical Systems 107-81, California Institute of Technology, Pasadena, CA 91125, email: marsden@cds.caltech.edu
} 


\section{Contents}

1 Introduction $\quad 2$

2 The Convergence of 3-Dimensional Elasticity to Shells 6

2.1 Three Dimensional Elastic Bodies . . . . . . . . . . . . . . . . . . . 6

2.2 Unconstrained Elastic Shells . . . . . . . . . . . . . . . . . . . . 7

2.3 Approximation of Poisson Brackets . . . . . . . . . . . . . . . . . 9

2.4 An Almost-Poisson Embedding for the Unconstrained Shell . . . . . . . . . . . . . . 12

2.5 The Role of Boundary Conditions in the Limiting Equations . . . . . . . . . . . . . . . 12

2.6 A Simple Example: an Unconstrained Wave Equation . . . . . . . . . . . . . 16

2.7 Unconstrained Limiting Models . . . . . . . . . . . . . . . . . . . . 17

3 Constrained-director Shell Models $\quad 18$

3.1 The Limit of the Hamiltonian Structure . . . . . . . . . . . . . . . . . . 18

3.2 The Wave Equation as a Constrained Director Model . . . . . . . . . . . . . . . . . 20

4 The Kirchhoff Shell as a Limit of a 3d Saint Venant-Kirchhoff Material 23

4.1 Saint Venant-Kirchhoff Materials . . . . . . . . . . . . . . . . . . . . 23

4.2 The Kirchhoff Membrane Model . . . . . . . . . . . . . . . . . . . . . . . . 24

4.3 The Inextensible Kirchhoff Shell . . . . . . . . . . . . . . . . . . . . . . . . . . 24

5 The Convergence of 3d Elasticity to Rod Models 26

5.1 Limits of Hamiltonian Structures . . . . . . . . . . . . . . . . . 26

5.2 Constrained and Unconstrained Limiting Models . . . . . . . . . . . . . . . . 28

6 The Kirchhoff Elastica as a Limit of a 3d Saint Venant-Kirchhoff Material 30

6.1 Introduction . . . . . . . . . . . . . . . . . . . 30

6.2 A Limiting Director Model for Rods . . . . . . . . . . . . . . . . . . . . . . 30

6.3 An Inextensible Elastica with a Director . . . . . . . . . . . . . . . . . . . . . 32

6.4 The Euler-Kirchhoff Elastica . . . . . . . . . . . . . . . . . 35

\section{Introduction}

\section{The Goals and Main Results}

This paper studies the problem of convergence of three dimensional elasticity models to corresponding models for shells and rods. In our approach we make use of the Hamiltonian structure as a crucial tool in the analysis.

In the case of shells, we show that the Poisson bracket (applied to certain classes of functions) for a 3d elastic body converges to the Poisson bracket for a shell model described by a one-director Cosserat surface when the thickness goes to zero. Alternatively, we prove, in a sense made precise later, that there is an almost-Poisson embedding from the phase space of the shell to the phase space of the $3 \mathrm{~d}$ elastic body. We also establish the sense in which the Hamiltonians themselves converge. Taken together, this is what we mean by the convergence of Hamiltonian structures. This convergence implies that, in a certain weak sense, the dynamics of the elastic body is close 
to that of the shell. This provides one justification of the approximation of a thin body by the Cosserat surface in full generality, at least for time-evolution problems.

Starting with the Saint Venant-Kirchhoff constitutive model as a specific case to illustrate the general theory, we derive by our systematic procedure a Kirchhoff shell model in which the stored energy function is an explicit function of the mean and Gaussian curvature of the shell (see $\S 4$ ).

Our method also applies to the case of thin rods, as we show in $\S 5$ and $\S 6$. In particular, we are able to get rather large classes of geometrically exact rod models. In particular, starting with the Saint Venant-Kirchhoff constitutive model, we derive the Euler-Kirchhoff model for an elastica by our procedures without making any intermediate ad hoc hypotheses.

\section{Related Works and Background}

The problem of convergence of the Hamiltonian structure for a $3 \mathrm{~d}$ ideal fluid with a free boundary to that for the shallow water equation was considered in Ge, Kruse, Marsden and Scovel [1995]. The approach in the present paper is an outgrowth of this previous work. That paper also discusses the general setting of the problem of convergence of Hamiltonian structures and elaborates on the meaning of the weak convergence. The main difficulty that paper solved was how to deal with the incompressibility constraint, whereas in this paper, the main analytic complication we overcome is how to deal with the limiting form of the constitutive relation. We do not consider the incompressible case here, although it can presumably be done by combining the two approaches.

Traditionally, there were two methods to derive an approximation of a thin elastic body (see, for example, Antman $[1972,1994])$. The first one is using asymptotic analysis, which usually consists of expanding the solution and the equations using powers of the thickness of the plate. This has been applied to special models (cf. Ciarlet and Miara [1992]). A remarkable recent result is that of Fox, Raoult and Simo [1993] who showed that for the Saint Venant-Kirchhoff material, the membrane model, the inextensional model, and the von Kármán model are all limits of 3d elasticity as the thickness $2 \epsilon \rightarrow 0$ by choosing different orders of dependence on $\epsilon$ for various components of the loading. It is of interest to generalize these ideas to materials with more general constitutive relations.

The second method, sometimes called the projection-constraint method (cf. Antman [1972]), is a Galerkin type method and has been extensively used. For example, the shell model described by a one-director Cosserat surface can be derived by such a method. However, this method is not easy to justify by means of an asymptotic analysis.

More recently, the theory of $\Gamma$-convergence has been applied successfully to time-independent problems in elasticity in Le Dret and Raoult [1995]. It would be of interest to see if these ideas can be applied to time-evolutionary problems.

We should mention explicitly that while we do examine the formal asymptotics of the problem and tie this with the Hamiltonian structure, we do not attempt to prove here the strong convergence of solutions in any sense. However, we think that the techniques of Marsden, Ratiu and Raugel [1995] will be useful in this problem and this will be a subject of a future investigation.

\section{The Approach in the Present Paper}

We take a different approach than previous authors, namely to study the convergence of the Poisson bracket, or alternatively to show that the natural embedding from the phase space of the Cosserat 
surface to that of a thin body is an almost-Poisson map. One reason for this approach is that we are mainly concerned with the time evolution problem, not with the equilibrium problem.

Poisson brackets are a useful tool for studying evolution problems since the equations of motion can be written in Poisson bracket form (see, for example, Marsden and Hughes [1994], Simo, Marsden and Krishnaprasad [1988]). Including them in the asymptotic analysis represents one of our main contributions augmenting previous approaches. We should also mention that the approach to asymptotics by incorporating Hamiltonian structures was central to the important work of Camassa and Holm [1993] and related papers.

Our method combines Hamiltonian structures with the projection-constraint method and asymptotics. In particular, we prove convergence of the equations in a weak sense using a calculation similar to that in the projection method. Our method incorporates the boundary conditions into the Hamiltonian structure in a natural way (as in the so-called natural boundary conditions in variational problems) rather than applying (rescaled) forces. Also, we can work either with the original domain or we can rescale the thin domain into one which is independent of $\epsilon$. John [1971] dealt with plates having no loadings and periodic boundary conditions in the plate directions. We note that the papers of Antman and Warner [1967] and John [1971] work with a thin domain that is not rescaled, while most other authors that deal with the asymptotics do rescale the domain. For us, we can do it either with or without rescaling and our experience is that retaining this flexibility is quite useful.

The kind of rescaling done determines, in part, the model that one gets in the limit. One of our main points is that all of this structure can be seen as various assumptions on the Hamiltonian structure. In general terms, we can divide the theories one gets as being constrained or unconstrained. Here, constrained means that there is a relation determined between the director variables and the position variables. In other approaches, the assumptions on the asymptotics that are needed to get the various theories are achieved by means of different scalings in different variables and in the external loadings, cf. Fox, Raoult and Simo [1993]. In our approach one sees the same thing by simple assumptions on the scalings that appear directly in the Hamiltonian. In particular, with a simple assumption on the scaling of the director variables, one gets the general unconstrained director theory.

\section{The Set up for Shells}

For the case of shells, we consider the motion of a thin elastic body with a three dimensional reference configuration of the form ${ }^{1}$

$$
D_{\epsilon}:=\Omega \times[-\epsilon, \epsilon],
$$

where $\Omega$ is a bounded domain in $\mathbb{R}^{2}$. We let $x$ denote the variables in the domain $\Omega$ and let $y$ denote the variable in the transverse direction, so that $-\epsilon \leq y \leq \epsilon$. Let $\phi$ denote a configuration of the body, that is, $\phi$ is a map of $D_{\epsilon}$ to $\mathbb{R}^{3}$ and we consider a three dimensional Hamiltonian of the form

$$
H(\phi, \dot{\phi}, \epsilon):=\iiint_{D_{\epsilon}}\left(\frac{1}{2} \dot{\phi} \cdot \dot{\phi}+g\left(x, y, \phi, \phi_{x}, \phi_{y}, \epsilon\right)\right) d^{2} x d y
$$

\footnotetext{
${ }^{1}$ Even though this reference configuration has a flat midsurface, we will shortly be taking approximations about a nontrivial configuration with a nonflat midsurface. Thus, we are considering shells here and not necessarily the special case of plates.
} 
where "." stands for the standard inner product in $\mathbb{R}^{3}$ and the overdot denotes the time derivative. We write $d^{2} x d y=d V$ for the standard Euclidean volume element. The equations of motion are given by Hamilton's equations for this Hamiltonian, or equivalently, the Euler-Lagrange equations for the corresponding Lagrangian (see, for example, Marsden and Hughes [1994]). Let $v:=\phi_{y}$. We will show that if

$$
\frac{\partial g}{\partial v}=O\left(\epsilon^{2}\right)
$$

then the limiting $2 \mathrm{~d}$ system as $\epsilon \rightarrow 0$ is an unconstrained Cosserat-model. Notice that the $3 \mathrm{~d}$ model is in this case necessarily anisotropic. Otherwise, the limiting process will lead in general to constraints on the director and the displacement; that is, the limiting system is a constrained director model. Under the additional assumption that $\partial^{2} g / \partial v^{2}$ does not vanish anywhere, we can use the implicit function theorem to express the director field as a function of the displacement field. The 2d shell models studied in Ciarlet and Lods [1994], Ciarlet, Lods and Miara [1994] and Ciarlet [1994] fall into this category.

We show that there is an almost-Poisson map from the phase space of the constrained-director model to the phase space of the unconstrained director model. Thus, both the unconstrained one-director model and the constrained-director model are compatible.

The Hamiltonian structure, namely the Poisson bracket, the energy and the conserved quantities, has also been important in the long time computation of conservative systems (cf. Ge and Marsden [1988], Ge [1990], Ge and Scovel [1994]). Work of Simo and his collaborators already shows that the Hamiltonian structure plays an important role in the numerical computation of the dynamics of rods and shells (compare Simo et al. [1992]). This structure has also been important in stability theory for elasticity; see for example, Simo, Posbergh and Marsden [1990, 1991] and Maddocks [1984, 1991]. We also note that Foltinek [1994] shows how to get integrals for the equations of the Euler elastica using symmetry and momentum maps.

As an application of the methods developed in this paper, we derive the membrane model and various inextensional models (for shells and rods) from the 3d Saint Venant-Kirchhoff model.

\section{Organization of the Paper}

In $\S 2$ we discuss the relation between the canonical 3d Poisson bracket and an $\epsilon$-dependent $2 \mathrm{~d}$ bracket for one-director shells, where, as above, $2 \epsilon$ is the shell thickness. The results in this section are used later in the paper to establish the limit theorems as the thickness tends to zero. In particular, in $\S 2.4$ we show that the natural embedding from the phase space of the one-director shell model to the $3 \mathrm{~d}$ model is an almost-Poisson embedding. In $\S 2.5$ we discuss the boundary conditions through a study of the dynamics of the limiting $2 \mathrm{~d}$ shell model. An example of unconstrained director models is discussed.

In $\S 3$ we introduce the constrained-director model for shells and show that the natural embedding from the phase space of the constrained-director model to that of the one-director model is almost-Poisson. In $§ 3.2$ we give several examples of constrained-director models. In particular, for linear plate theory, we obtain the membrane model (Ciarlet [1994]) as the constrained-director model. We also show how a geometrically exact shell model can be obtained as the limit of a 3d Saint Venant-Kirchhoff material.

In $\S 4$ we show how to derive the Kirchhoff shell as a limit of a 3d Saint Venant-Kirchhoff material. We discuss how constraints that arise from the limiting procedures enter into the models 
as well as other constraints such as inextensibility that are imposed as holonomic constraints.

In $\S 5, \S 6$ we discuss most of these same aspects for rod models. In particular, we show how to obtain the Kirchhoff elastic rod model as a limit of a 3d Saint Venant-Kirchhoff material.

\section{Remarks on Notation}

For shells, we use $x$ to denote a pair of variables, say $\left(x_{1}, x_{2}\right)$, which parametrize the reference middle surface of the shell, and $y$ for a variable transverse to the shell. The placement field for the corresponding three dimensional elastic body is denoted $\phi$ and that for the middle surface of the shell is denoted $\varphi$. The director is denoted by $w$.

For rods, we use $z$ to denote the variables which parametrizes the reference central line of the rod, and $x, y$ for variables transverse to the rod. The placement field for the corresponding three dimensional elastic body is denoted $\phi$ and that for the central line of the rod is denoted $\varphi$. The directors are denoted by $w_{1}$ and $w_{2}$.

\section{The Convergence of 3-Dimensional Elasticity to Shells}

\subsection{Three Dimensional Elastic Bodies}

With the reference configuration $D_{\epsilon}$ as defined by (1.0.1) above, we introduce the further notation

$$
\begin{aligned}
\Omega_{u} & :=\Omega \times\{\epsilon\}, \\
\Omega_{l} & :=\Omega \times\{-\epsilon\}, \\
\Omega_{s} & :=\partial \Omega \times[-\epsilon, \epsilon],
\end{aligned}
$$

which denote the upper, lower and lateral boundary of $D_{\epsilon}$, respectively. The material (or Lagrangian) configuration space of the body is

$$
\mathcal{M}_{3 d}^{\epsilon}:=\left\{\phi \mid \phi: \Omega \times[-\epsilon, \epsilon] \rightarrow \mathbb{R}^{3} \text { is an embedding }\right\} .
$$

At the moment we leave the precise smoothness class in this definition and those to follow unspecified. In a specific context these can be dealt with as in Marsden and Hughes [1994]; we hope to return to these points in connection with existence theory in a later paper. The Lagrangian phase space is the tangent bundle of $\mathcal{M}_{3 d}^{\epsilon}$, namely

$$
T \mathcal{M}_{3 d}^{\epsilon}=\left\{(\phi, \dot{\phi}) \mid \phi \in \mathcal{M}_{3 d}^{\epsilon} \text { and } \dot{\phi}: \Omega \times[-\epsilon, \epsilon] \rightarrow \mathbb{R}^{3}\right\}
$$

On $\mathcal{M}_{3 d}^{\epsilon}$ we consider the Riemannian metric defined by

$$
\left\langle\left\langle\left(\phi, \dot{\phi}_{1}\right),\left(\phi, \dot{\phi}_{2}\right)\right\rangle\right\rangle_{3 d}^{\epsilon}=\iiint_{D_{\epsilon}} \dot{\phi}_{1} \cdot \dot{\phi}_{2} d V
$$

We identify $T^{*} \mathcal{M}_{3 d}^{\epsilon}$ and $T \mathcal{M}_{3 d}^{\epsilon}$ via this metric. We will use the standard canonical cotangent bracket for functionals $F_{1}, F_{2}$ on $T^{*} \mathcal{M}_{3 d}^{\epsilon}$ and denote it by $\left\{F_{1}, F_{2}\right\}_{3 \mathrm{~d} \text { can }}^{\epsilon}$. Note that this depends on $\epsilon$ since the domain does. The induced bracket on $T \mathcal{M}_{3 d}^{\epsilon}$ obtained via the Legendre transformation is denoted $\left\{F_{1}, F_{2}\right\}_{3 \mathrm{~d}}^{\epsilon}$. 
In this section we consider hyperelastic materials whose corresponding energy functionals are defined on $T \mathcal{M}_{3 d}^{\epsilon}$ of the form

$$
\begin{aligned}
F= & \iint_{\Omega} \int_{-\epsilon}^{\epsilon} f\left(x, y, \phi, \partial_{x} \phi, \partial_{y} \phi, \dot{\phi}, \epsilon\right) d^{2} x d y+\iint_{\Omega_{u}} \epsilon f_{u}\left(x, \phi, \partial_{x} \phi, \partial_{y} \phi, \epsilon\right) d^{2} x \\
& +\iint_{\Omega_{l}} \epsilon f_{l}\left(x, \phi, \partial_{x} \phi, \partial_{y} \phi, \epsilon\right) d^{2} x+\iint_{\Omega_{s}} f_{s}\left(s_{1}, y, \phi, \partial_{s} \phi, \partial_{y} \phi, \epsilon\right) d A
\end{aligned}
$$

where $s=\left(s_{1}, s_{2}\right)$ defines coordinates in a neighborhood of the curve $\partial \Omega$, such that the curve itself is parametrized by $s_{1}$, and $d A$ is the area element induced on $\Omega_{s}$. The functions $f, f_{u}, f_{l}, f_{s}$ depend on $\epsilon$ as a parameter generally. Note that, in particular, the elastic energy is of this form, where the first term corresponds to the stored energy and the body force, and the remaining terms correspond to energy terms giving rise to surface forces along the upper, lower, and lateral surfaces respectively.

\subsection{Unconstrained Elastic Shells}

Now we turn to the approximation by the one-director model described by a Cosserat surface in the limit $\epsilon \rightarrow 0$. Intuitively, when $y \in[-\epsilon, \epsilon]$ is small, a configuration $\phi(x, y)$ can be expanded in powers of $y$ :

$$
\phi(x, y)=\phi(x, 0)+\frac{\partial \phi}{\partial y}(x, 0) y+O\left(y^{2}\right)
$$

Denote

$$
\varphi(x):=\phi(x, 0), \text { and } w(x):=\frac{\partial \phi}{\partial y}(x, 0) .
$$

For the approximation, we consider the space of maps affine in $y$, namely those of the form $\varphi(x)+$ $y w(x)$, which we can identify with the space of pairs

$$
\mathcal{M}_{2 d}=\left\{(\varphi, w) \mid \varphi: \Omega \rightarrow \mathbb{R}^{3} \text { is an embedding and } w: \Omega \rightarrow \mathbb{R}^{3}\right\} .
$$

Here $w$ is usually called the director field, as in Naghdi [1972]. The tangent bundle of $\mathcal{M}_{2 d}$ is given by

$$
T \mathcal{M}_{2 d}=\left\{(\varphi, w, \dot{\varphi}, \dot{w}) \mid(\varphi, w) \in \mathcal{M}_{2 d} \text { and } \dot{\varphi}, \dot{w}: \Omega \rightarrow \mathbb{R}^{3}\right\} .
$$

If we take a higher order truncation of $\phi$, we obtain a model with more than one director field. As we shall see, an appropriate $\epsilon$-dependent Poisson bracket on this space will be an approximation to the $3 \mathrm{~d}$ Poisson bracket. When one is dealing with the Hamiltonian formulation of elasticity, one must include boundary conditions in a standard way (see John [1971] and Marsden and Hughes [1994]). Those conditions do not play an important role in this section but will be important in Sections 3 and 4.

Define a map

$$
A_{\epsilon}: T \mathcal{M}_{2 d} \rightarrow T \mathcal{M}_{3 d}^{\epsilon}
$$

by

$$
A_{\epsilon}(\varphi(x), w(x), \dot{\varphi}(x), \dot{w}(x)) \rightarrow(\varphi(x)+y w(x), \dot{\varphi}(x)+y \dot{w}(x)), \quad y \in[-\epsilon, \epsilon]
$$


If we are given a functional of the form (2.1.2), which includes the energy for $3 \mathrm{~d}$ elasticity, the corresponding approximate functional is obtained by substituting $\phi(x, y)=\varphi(x)+y w(x), \dot{\phi}(x, y)=$ $\dot{\varphi}(x)+y \dot{w}(x)$ in $(2.1 .2)$, that is,

$$
\begin{aligned}
F \circ A_{\epsilon}= & \iint_{\Omega} \int_{-\epsilon}^{\epsilon} f\left(x, y, \varphi(x)+y w(x), \partial_{x} \varphi(x)+y \partial_{x} w(x), w(x), \dot{\varphi}(x)+y \dot{w}(x), \epsilon\right) d^{2} x d y \\
& +\iint_{\Omega_{u}} \epsilon f_{u}\left(x, \varphi(x)+\epsilon w(x), \partial_{x} \varphi(x)+\epsilon \partial_{x} w(x), w(x)\right) d^{2} x \\
& +\iint_{\Omega_{l}} \epsilon f_{l}\left(x, \varphi(x)-\epsilon w(x), \partial_{x} \varphi(x)-\epsilon \partial_{x} w(x), w(x)\right) d^{2} x \\
& +\iint_{\Omega_{s}} f_{s}\left(s_{1}, y, \varphi\left(s_{1}\right)+y w\left(s_{1}\right), \partial_{s} \varphi\left(s_{1}\right)+y \partial_{s} w\left(s_{1}\right), w\left(s_{1}\right)\right) d A
\end{aligned}
$$

For example, letting $U=\dot{\varphi}+y \dot{w}$, the approximate functional associated with the kinetic energy functional is given by the induced kinetic energy functional

$$
\frac{1}{2}\left\langle\langle(\dot{\varphi}, \dot{w}),(\dot{\varphi}, \dot{w})\rangle_{2 \mathrm{~d}}^{\epsilon}:=\frac{1}{2}\langle\langle U, U\rangle\rangle_{3 d}^{\epsilon}=\iint_{\Omega}\left(\epsilon \dot{\varphi} \cdot \dot{\varphi}+\frac{\epsilon^{3}}{3} \dot{w} \cdot \dot{w}\right) d^{2} x .\right.
$$

Now we compute the Poisson bracket for $2 \mathrm{~d}$ elasticity. First we identify $T \mathcal{M}_{2 d}$ with $T^{*} \mathcal{M}_{2 d}$ via the Riemannian metric (2.2.1) and obtain the $\epsilon$-dependent Legendre transformation

$$
p_{1}=2 \epsilon \dot{\varphi}, p_{2}=\frac{2 \epsilon^{3}}{3} \dot{w}
$$

Here $p_{1}, p_{2}$ are the generalized conjugate momenta to $\varphi, w$ respectively. We use, as before, the canonical bracket on $T^{*} \mathcal{M}_{2 d}$ and will denote it by $\left\{F_{1}, F_{2}\right\}_{2 \mathrm{~d} \text { can }}$. The bracket induced on $T \mathcal{M}_{2 d}$ via the Legendre transform will be denoted by $\left\{F_{1}, F_{2}\right\}_{2 \mathrm{~d}}^{\epsilon}$. A short calculation shows that this Poisson bracket is given by

$$
\left\{F_{1}, F_{2}\right\}_{2 d}^{\epsilon}=\frac{1}{2 \epsilon} D_{\varphi} F_{1} \frac{\delta F_{2}}{\delta \dot{\varphi}}+\frac{3}{2 \epsilon^{3}} D_{w} F_{1} \frac{\delta F_{2}}{\delta \dot{w}}-\frac{1}{2 \epsilon} D_{\varphi} F_{2} \frac{\delta F_{1}}{\delta \dot{\varphi}}-\frac{3}{2 \epsilon^{3}} D_{w} F_{2} \frac{\delta F_{1}}{\delta \dot{w}} .
$$

Notice that this bracket contains singular terms of order $1 / \epsilon^{3}$. In particular, one cannot take the limit $\epsilon=0$.

A possible way to resolve this singularity is to introduce a change of variable $w \rightarrow \bar{w}=w \epsilon$, then in the rescaled coordinates the bracket has the form

$$
\left\{F_{1}, F_{2}\right\}_{2 d}^{\epsilon}=\frac{1}{2 \epsilon} D_{\varphi} F_{1} \frac{\delta F_{2}}{\delta \dot{\varphi}}+\frac{3}{2 \epsilon} D_{\bar{w}} F_{1} \frac{\delta F_{2}}{\delta \dot{\bar{w}}}-\frac{1}{2 \epsilon} D_{\varphi} F_{2} \frac{\delta F_{1}}{\delta \dot{\varphi}}-\frac{3}{2 \epsilon} D_{\bar{w}} F_{2} \frac{\delta F_{1}}{\delta \dot{\bar{w}}} .
$$

This change of coordinates corresponds to a change of variables $y \mapsto \bar{y}=y / \epsilon$ in the reference configuration of the 3d-model. After this rescaling, the singularity in the Poisson tensor has been resolved (up to an overall scale factor). However, the Hamiltonian will have the following form

$$
\iint_{\Omega}\left[f\left(x, 0, \varphi, \frac{\partial \varphi}{\partial x}, \frac{1}{\epsilon} \bar{w}, \frac{1}{\epsilon} \dot{\bar{w}}\right)+O(\epsilon)\right] d^{2} x
$$

which in general does not converge as $\epsilon \rightarrow 0$ unless one makes special assumptions on the behavior of the function $f$ "at infinity". In $§ 2.6$ we will consider Hamiltonians that do converge after rescaling 
as $\epsilon \rightarrow 0$, and restriction of the Poisson bracket to such functions leads to unconstrained-director models.

Another way to eliminate the singularity is to introduce holonomic constraints on the director vector field, which is the contents of $\S 3$.

\subsection{Approximation of Poisson Brackets}

Let $F_{i}, i=1,2$ be two functionals of the form (2.1.2). In this section we prove the following theorem:

Theorem 2.1 Let $F_{1}$ and $F_{2}$ be two functionals of the form (2.1.2) and $F_{i} \circ A_{\epsilon}$ their pull backs via the embedding $A_{\epsilon}$, which we will call the approximation embedding. Let $(\phi, \dot{\phi}) \in T \mathcal{M}_{3 d}^{\epsilon}$, and let $\varphi(x)=\phi(x, 0), w=\frac{\partial}{\partial y} \phi(x, 0), \dot{\varphi}(x)=\dot{\phi}(x, 0), \dot{w}=\frac{\partial}{\partial y} \dot{\phi}(x, 0)$. Then

$$
\left\{F_{1}, F_{2}\right\}_{3 d}^{\epsilon}(\phi, \dot{\phi})=\left\{F_{1} \circ A_{\epsilon}, F_{2} \circ A_{\epsilon}\right\}_{2 d}^{\epsilon}(\varphi, w, \dot{\varphi}, \dot{w})+O\left(\epsilon^{3}\right)
$$

Proof The derivatives of $F_{1}$ and $F_{2}$ are (the index $i$ is omitted for the moment)

$$
\begin{aligned}
D_{\phi} F \cdot \delta \phi= & \iiint_{\Omega \times[-\epsilon, \epsilon]}\left(\frac{\partial f}{\partial \phi} \delta \phi+\frac{\partial f}{\partial u} \partial_{x} \delta \phi+\frac{\partial f}{\partial v} \partial_{y} \delta \phi\right) d^{2} x d y \\
& +\epsilon \iint_{\Omega_{u}}\left(\frac{\partial f_{u}}{\partial \phi} \delta \phi+\frac{\partial f_{u}}{\partial u} \partial_{x} \delta \phi+\frac{\partial f_{u}}{\partial v} \partial_{y} \delta \phi\right) d^{2} x \\
& +\epsilon \iint_{\Omega_{l}}\left(\frac{\partial f_{l}}{\partial \phi} \delta \phi+\frac{\partial f_{l}}{\partial u} \partial_{x} \delta \phi+\frac{\partial f_{l}}{\partial v} \partial_{y} \delta \phi\right) d^{2} x \\
& +\iint_{\Omega_{s}}\left(\frac{\partial f_{s}}{\partial \phi} \delta \phi+\frac{\partial f_{s}}{\partial m} \partial_{s} \delta \phi+\frac{\partial f_{s}}{\partial v} \partial_{y} \delta \phi\right) d A
\end{aligned}
$$

where $u=\partial_{x} \phi, v=\partial_{y} \phi, m=\partial_{s} \phi$, and

$$
D_{\dot{\phi}} F \cdot \delta \dot{\phi}=\iiint_{\Omega \times[-\epsilon, \epsilon]}\left(\frac{\partial f}{\partial \dot{\phi}} \cdot \delta \dot{\phi}\right) d^{2} x d y .
$$


Thus, the $3 \mathrm{~d}$ bracket is given by

$$
\begin{aligned}
\left\{F_{1}, F_{2}\right\}_{3 d}^{\epsilon}= & \iiint_{\Omega \times[-\epsilon, \epsilon]}\left(\frac{\partial f_{1}}{\partial \phi} \frac{\partial f_{2}}{\partial \dot{\phi}}+\frac{\partial f_{1}}{\partial u} \partial_{x} \frac{\partial f_{2}}{\partial \dot{\phi}}+\frac{\partial f_{1}}{\partial v} \partial_{y} \frac{\partial f_{2}}{\partial \dot{\phi}}\right) d^{2} x d y \\
& -\iiint_{\Omega \times[-\epsilon, \epsilon]}\left(\frac{\partial f_{2}}{\partial \phi} \frac{\partial f_{1}}{\partial \dot{\phi}}+\frac{\partial f_{2}}{\partial u} \partial_{x} \frac{\partial f_{1}}{\partial \dot{\phi}}+\frac{\partial f_{2}}{\partial v} \partial_{y} \frac{\partial f_{1}}{\partial \dot{\phi}}\right) d^{2} x d y \\
& +\epsilon \iint_{\Omega_{u}}\left(\frac{\partial f_{1, u}}{\partial \phi} \frac{\partial f_{2}}{\partial \dot{\phi}}+\frac{\partial f_{1, u}}{\partial u} \partial_{x} \frac{\partial f_{2}}{\partial \dot{\phi}}+\frac{\partial f_{1, u}}{\partial v} \partial_{y} \frac{\partial f_{2}}{\partial \dot{\phi}}\right) d^{2} x \\
& -\epsilon \iint_{\Omega_{u}}\left(\frac{\partial f_{2, u}}{\partial \phi} \frac{\partial f_{1}}{\partial \dot{\phi}}+\frac{\partial f_{2, u}}{\partial u} \partial_{x} \frac{\partial f_{1}}{\partial \dot{\phi}}+\frac{\partial f_{2, u}}{\partial v} \partial_{y} \frac{\partial f_{1}}{\partial \dot{\phi}}\right) d^{2} x \\
& +\epsilon \iint_{\Omega_{l}}\left(\frac{\partial f_{1, l}}{\partial \phi} \frac{\partial f_{2}}{\partial \dot{\phi}}+\frac{\partial f_{1, l}}{\partial u} \partial_{x} \frac{\partial f_{2}}{\partial \dot{\phi}}+\frac{\partial f_{1, l}}{\partial v} \partial_{y} \frac{\partial f_{2}}{\partial \dot{\phi}}\right) d^{2} x \\
& -\epsilon \iint_{\Omega_{l}}\left(\frac{\partial f_{2, l}}{\partial \phi} \frac{\partial f_{1}}{\partial \dot{\phi}}+\frac{\partial f_{2, l}}{\partial u} \partial_{x} \frac{\partial f_{1}}{\partial \dot{\phi}}+\frac{\partial f_{2, l}}{\partial v} \partial_{y} \frac{\partial f_{1}}{\partial \dot{\phi}}\right) d^{2} x \\
& +\iint_{\Omega_{s}}\left(\frac{\partial f_{1, s}}{\partial \phi} \frac{\partial f_{2}}{\partial \dot{\phi}}+\frac{\partial f_{1, s}}{\partial m} \partial_{s} \frac{\partial f_{2}}{\partial \dot{\phi}}+\frac{\partial f_{1, s}}{\partial v} \partial_{y} \frac{\partial f_{2}}{\partial \dot{\phi}}\right) d A \\
& -\iint_{\Omega_{s}}\left(\frac{\partial f_{2, s}}{\partial \phi} \frac{\partial f_{1}}{\partial \dot{\phi}}+\frac{\partial f_{2, s}}{\partial m} \partial_{s} \frac{\partial f_{1}}{\partial \dot{\phi}}+\frac{\partial f_{2, s}}{\partial v} \partial_{y} \frac{\partial f_{1}}{\partial \dot{\phi}}\right) d A .
\end{aligned}
$$

In the integrand we expand the solution in powers of $y$. First note that after making use of the equality $\phi=\varphi+y w+O\left(y^{2}\right)$, we have

$$
\partial_{y} \frac{\partial f}{\partial \dot{\phi}}=\frac{\partial^{2} f}{\partial y \partial \dot{\phi}}+\frac{\partial^{2} f}{\partial \phi \partial \dot{\phi}} w+\frac{\partial^{2} f}{\partial u \partial \dot{\phi}} \partial_{x} w+\frac{\partial^{2} f}{\partial \dot{\phi}^{2}} \dot{w}+O(y) .
$$


Thus, the Poisson bracket (2.3.1) becomes

$$
\begin{aligned}
\left\{F_{1}, F_{2}\right\}_{3 d}^{\epsilon} & =2 \epsilon \iint_{\Omega}\left[\frac{\partial f_{1}}{\partial \phi} \frac{\partial f_{2}}{\partial \dot{\phi}}+\frac{\partial f_{1}}{\partial u} \partial_{x} \frac{\partial f_{2}}{\partial \dot{\phi}}+\frac{\partial f_{1}}{\partial v}\left(\frac{\partial^{2} f_{2}}{\partial y \partial \dot{\phi}}+\frac{\partial^{2} f_{2}}{\partial \phi \partial \dot{\phi}} w+\frac{\partial^{2} f_{2}}{\partial u \partial \dot{\phi}} \partial_{x} w+\frac{\partial^{2} f_{2}}{\partial \dot{\phi}^{2}} \dot{w}\right)\right] d^{2} x \\
& -2 \epsilon \iint_{\Omega}\left[\frac{\partial f_{2}}{\partial \phi} \frac{\partial f_{1}}{\partial \dot{\phi}}+\frac{\partial f_{2}}{\partial u} \partial_{x} \frac{\partial f_{1}}{\partial \dot{\phi}}+\frac{\partial f_{2}}{\partial v}\left(\frac{\partial^{2} f_{1}}{\partial y \partial \dot{\phi}}+\frac{\partial^{2} f_{1}}{\partial \phi \partial \dot{\phi}} w+\frac{\partial^{2} f_{1}}{\partial u \partial \dot{\phi}} \partial_{x} w+\frac{\partial^{2} f_{2}}{\partial \dot{\phi}^{2}} \dot{w}\right)\right] d^{2} x \\
& +\epsilon \iint_{\Omega}\left[\frac{\partial f_{1, u}}{\partial \phi} \frac{\partial f_{2}}{\partial \dot{\phi}}+\frac{\partial f_{1, u}}{\partial u} \partial_{x} \frac{\partial f_{2}}{\partial \dot{\phi}}+\frac{\partial f_{1, u}}{\partial v}\left(\frac{\partial^{2} f_{2}}{\partial y \partial \dot{\phi}}+\frac{\partial^{2} f_{2}}{\partial \phi \partial \dot{\phi}} w+\frac{\partial^{2} f_{2}}{\partial u \partial \dot{\phi}} \partial_{x} w+\frac{\partial^{2} f_{2}}{\partial \dot{\phi}^{2}} \dot{w}\right)\right] d^{2} x \\
& -\epsilon \iint_{\Omega}\left[\frac{\partial f_{2, u}}{\partial \phi} \frac{\partial f_{1}}{\partial \dot{\phi}}+\frac{\partial f_{2, u}}{\partial u} \partial_{x} \frac{\partial f_{1}}{\partial \dot{\phi}}+\frac{\partial f_{2, u}}{\partial v}\left(\frac{\partial^{2} f_{1}}{\partial y \partial \dot{\phi}}+\frac{\partial^{2} f_{1}}{\partial \phi \partial \dot{\phi}} w+\frac{\partial^{2} f_{1}}{\partial u \partial \dot{\phi}} \partial_{x} w+\frac{\partial^{2} f_{2}}{\partial \dot{\phi}^{2}} \dot{w}\right)\right] d^{2} x \\
& +\epsilon \iint_{\Omega}\left[\frac{\partial f_{1, l}}{\partial \phi} \frac{\partial f_{2}}{\partial \dot{\phi}}+\frac{\partial f_{1, l}}{\partial u} \partial_{x} \frac{\partial f_{2}}{\partial \dot{\phi}}+\frac{\partial f_{1, l}}{\partial v}\left(\frac{\partial^{2} f_{2}}{\partial y \partial \dot{\phi}}+\frac{\partial^{2} f_{2}}{\partial \phi \partial \dot{\phi}} w+\frac{\partial^{2} f_{2}}{\partial u \partial \dot{\phi}} \partial_{x} w+\frac{\partial^{2} f_{2}}{\partial \dot{\phi}^{2}} \dot{w}\right)\right] d^{2} x \\
& -\epsilon \iint_{\Omega}\left[\frac{\partial f_{2, l}}{\partial \phi} \frac{\partial f_{1}}{\partial \dot{\phi}}+\frac{\partial f_{2, l}}{\partial u} \partial_{x} \frac{\partial f_{1}}{\partial \dot{\phi}}+\frac{\partial f_{2, l}}{\partial v}\left(\frac{\partial^{2} f_{1}}{\partial y \partial \dot{\phi}}+\frac{\partial^{2} f_{1}}{\partial \phi \partial \dot{\phi}} w+\frac{\partial^{2} f_{1}}{\partial u \partial \dot{\phi}} \partial_{x} w+\frac{\partial^{2} f_{1}}{\partial \dot{\phi}^{2}} \dot{w}\right)\right] d^{2} x \\
& +2 \epsilon \iint_{\partial \Omega}\left[\frac{\partial f_{1, s}}{\partial \phi} \frac{\partial f_{2}}{\partial \dot{\phi}}+\frac{\partial f_{1, s}}{\partial m} \partial_{s} \frac{\partial f_{2}}{\partial \dot{\phi}}+\frac{\partial f_{1, s}}{\partial v}\left(\frac{\partial^{2} f_{2}}{\partial y \partial \dot{\phi}}+\frac{\partial^{2} f_{2}}{\partial \phi \partial \dot{\phi}} w+\frac{\partial^{2} f_{2}}{\partial u \partial \dot{\phi}} \partial_{x} w+\frac{\partial^{2} f_{2}}{\partial \dot{\phi}^{2}} \dot{w}\right)\right] d s_{1} \\
& -2 \epsilon \int_{\partial \Omega}\left[\frac{\partial f_{2, s}}{\partial \phi} \frac{\partial f_{1}}{\partial \dot{\phi}}+\frac{\partial f_{2, s}}{\partial m} \partial_{s} \frac{\partial f_{1}}{\partial \dot{\phi}}+\frac{\partial f_{2, s}}{\partial v}\left(\frac{\partial^{2} f_{1}}{\partial y \partial \dot{\phi}}+\frac{\partial^{2} f_{1}}{\partial \phi \partial \dot{\phi}} w+\frac{\partial^{2} f_{1}}{\partial u \partial \dot{\phi}} \partial_{x} w+\frac{\partial^{2} f_{1}}{\partial \dot{\phi}^{2}} \dot{w}\right)\right] d s_{1} \\
+ & O\left(\epsilon^{3}\right),
\end{aligned}
$$

where the functions are evaluated at $(x, y, \phi, u, v, \dot{\phi})=\left(x, 0, \varphi, \partial_{x} \varphi, w, \dot{\varphi}\right)$.

Next we compute the $2 \mathrm{~d}$ brackets of $F_{1} \circ A_{\epsilon}$ and $F_{2} \circ A_{\epsilon}$. For functions of the form introduced earlier, which includes the $2 \mathrm{~d}$ energy, we have

$$
\begin{aligned}
F \circ A_{\epsilon}= & 2 \epsilon \iint_{\Omega} f\left(x, 0, \varphi(x), \partial_{x} \varphi(x), w(x), \dot{\varphi}, \epsilon\right) d^{2} x+\epsilon \iint_{\Omega} f_{u}\left(x, \varphi, \partial_{x} \varphi, w\right) d^{2} x \\
& +\epsilon \iint_{\Omega} f_{l}\left(x, \varphi, \partial_{x} \varphi, w\right) d^{2} x+2 \epsilon \int_{\partial \Omega} f_{s}\left(s_{1}, 0, \varphi, \partial_{s} \varphi, w\right) d s_{1} \\
& + \text { terms of order } \epsilon^{2} \text { independent of } \dot{\phi} \text { and } \dot{w} \\
& +\frac{2 \epsilon^{3}}{3} \iint_{\Omega}\left[\left(\frac{\partial}{\partial y}+w \frac{\partial}{\partial \phi}+\partial_{x} w \frac{\partial}{\partial u}+\dot{w} \frac{\partial}{\partial \dot{\Phi}}\right)^{2} f\left(x, 0, \varphi, \partial_{x} \varphi, \dot{\varphi}\right)\right] d^{2} x+O\left(\epsilon^{5}\right) .
\end{aligned}
$$

Thus, we obtain an asymptotic formula

$$
\frac{\partial\left(F \circ A_{\epsilon}\right)}{\partial \dot{w}}=4 \frac{\epsilon^{3}}{3}\left(\frac{\partial}{\partial y}+w \frac{\partial}{\partial \phi}+\partial_{x} w \frac{\partial}{\partial u}+\dot{w} \frac{\partial}{\partial \dot{\phi}}\right) \frac{\partial}{\partial \dot{\phi}} f\left(x, 0, \varphi, \partial_{x} \varphi, w, \dot{\varphi}\right)+O\left(\epsilon^{5}\right) .
$$

Hence, we see that $\left\{F_{1} \circ A_{\epsilon}, F_{2} \circ A_{\epsilon}\right\}_{2 d}^{\epsilon}$ is equal to $\left\{F_{1}, F_{2}\right\}_{3 d}^{\epsilon}$ up to an error of $O\left(\epsilon^{3}\right)$. This proves the theorem.

Remark. If one rescales the domain from $\Omega \times[-\epsilon, \epsilon]$ to $\Omega \times[-1,1]$ by the change of variable $y=\epsilon \bar{y}$ then one should keep in mind that

$$
\frac{1}{\epsilon} \frac{\partial \phi}{\partial \bar{y}}=O(1)
$$


in the computations. This rescaling enables us to work with a $3 \mathrm{~d}$ reference configuration of fixed height. Our method of deriving the $2 \mathrm{~d}$ model carries over to this situation and leads to the same limiting system up to change of variables. This is not surprising because the Poisson bracket is a geometric concept independent of the choice of coordinates (i.e. variables).

\subsection{An Almost-Poisson Embedding for the Unconstrained Shell}

As a corollary of Theorem 2.1, we show that there is an almost-Poisson embedding from the phase space of the $2 \mathrm{~d}$ shell into that for the $3 \mathrm{~d}$ elastic body.

Recall that if we have two Poisson manifolds $P_{1}, P_{2}$ with Poisson brackets $\{\cdot, \cdot\}_{P_{1}},\{\cdot, \cdot\}_{P_{2}}$ respectively, then a map $A: P_{1} \rightarrow P_{2}$ is called a Poisson map if

$$
\left\{F_{1}, F_{2}\right\}_{P_{2}} \circ A=\left\{F_{1} \circ A, F_{2} \circ A\right\}_{P_{1}}
$$

for every pair of functions $F_{1}, F_{2}$ on $P_{2}$. If $A$ is a Poisson map, then every Hamiltonian dynamical system on $P_{2}$ can be pulled back to $P_{1}$. That is, if $\Theta_{F}^{t}$ and $\Theta_{F \circ A}^{t}$ denote the Hamiltonian flows of the Hamiltonians $F$ and $F \circ A$ respectively, then

$$
A \circ \Theta_{F \circ A}^{t}=\Theta_{F}^{t} \circ A .
$$

These concepts can be generalized to almost-Poisson embeddings. We say that $A$ is an $O(\epsilon)$ Poisson map if

$$
\left\{F_{1}, F_{2}\right\}_{P_{2}} \circ A=\left\{F_{1} \circ A, F_{2} \circ A\right\}_{P_{1}}+O(\epsilon) .
$$

In this case, we have the relation ( for a proof see Ge et al. [1994])

$$
A \circ \Theta_{F \circ A}^{t}=\Theta_{F}^{t} \circ A+O(\epsilon)
$$

interpreted in a suitable weak sense when one is dealing with infinite dimensional system, that is, with PDEs.

Now we apply these general ideas to the approximation of a thin elastic body by a shell. It follows from Theorem 2.1 that the map $A_{\epsilon}$ introduced above is an $O\left(\epsilon^{3}\right)$-Poisson embedding:

Corollary 2.2 For two functionals $F_{1}, F_{2}$ of the form (2.1.2), we have

$$
\left\{F_{1}, F_{2}\right\}_{3 d}^{\epsilon} \circ A_{\epsilon}=\left\{F_{1} \circ A_{\epsilon}, F_{2} \circ A_{\epsilon}\right\}_{2 d}^{\epsilon}+O\left(\epsilon^{3}\right) .
$$

\subsection{The Role of Boundary Conditions in the Limiting Equations}

Let $H: T \mathcal{M}_{3 d}^{\epsilon} \rightarrow \mathbb{R}$ be given, for example, as in the introduction. In this chapter we write down the differential equations corresponding to the equations

$$
\dot{F}=\{F, H\}_{3 d}^{\epsilon} \quad \text { for all } \quad F: T \mathcal{M}_{3 d}^{\epsilon} \rightarrow \mathbb{R},
$$

and

$$
\dot{F}=\left\{F, H \circ A_{\epsilon}\right\}_{2 d}^{\epsilon} \quad \text { for all } \quad F: T \mathcal{M}_{2 d}^{\epsilon} \rightarrow \mathbb{R},
$$

respectively. 
We are especially interested in the boundary conditions which are implied by (2.5.1) and (2.5.2) for the corresponding differential equations. These are natural boundary conditions to be distinguished from boundary conditions which are formulated as part of the definition of configuration space (e.g. conditions which say that certain parts of the boundary stay fixed throughout a motion).

To formulate the differential equations corresponding to (2.5.1) and (2.5.2) it is convenient to introduce functional derivatives, defined as follows:

The functional derivatives

$$
\begin{aligned}
\frac{\delta F}{\delta \phi}(\phi, \dot{\phi}): \Omega \times[-\epsilon, \epsilon] & \rightarrow \mathbb{R}^{3}, \\
\frac{\delta^{\prime} F}{\delta \phi}(\phi, \dot{\phi}): \partial(\Omega \times[-\epsilon, \epsilon]) & \rightarrow \mathbb{R}^{3}, \\
\frac{\delta F}{\delta \dot{\phi}}(\phi, \dot{\phi}): \Omega \times[-\epsilon, \epsilon] & \rightarrow \mathbb{R}^{3},
\end{aligned}
$$

of a function $F: T \mathcal{M}_{3 d}^{\epsilon} \rightarrow \mathbb{R}$ at a point $(\phi, \dot{\phi}) \in T \mathcal{M}_{3 d}^{\epsilon}$ are defined by

$$
\begin{aligned}
D_{\phi} F \cdot \delta \phi & =\iiint_{\Omega \times[-\epsilon, \epsilon]}\left(\frac{\delta F}{\delta \phi}(\phi, \dot{\phi}) \cdot \delta \phi\right) d^{2} x d y+\iint_{\partial(\Omega \times[-\epsilon, \epsilon])}\left(\frac{\delta^{\prime} F}{\delta \phi}(\phi, \dot{\phi}) \cdot \delta \phi\right) d A, \\
D_{\dot{\phi}} F \cdot \delta \dot{\phi} & =\iiint_{\Omega \times[-\epsilon, \epsilon]}\left(\frac{\delta F}{\delta \dot{\phi}}(\phi, \dot{\phi}) \cdot \delta \dot{\phi}\right) d^{2} x d y .
\end{aligned}
$$

Analogously we define the functional derivatives of a function $L: T \mathcal{M}_{2 d} \rightarrow \mathbb{R}$ at point $(\varphi, \dot{\varphi}, w, \dot{w}) \in$ $T \mathcal{M}_{2 d}$

$$
\begin{aligned}
\frac{\delta L}{\delta \varphi}(\varphi, \dot{\varphi}, w, \dot{w}): \Omega & \rightarrow \mathbb{R}^{3}, \\
\frac{\delta^{\prime} L}{\delta \varphi}(\varphi, \dot{\varphi}, w, \dot{w}): \partial \Omega & \rightarrow \mathbb{R}^{3}, \\
\frac{\delta L}{\delta \dot{\varphi}}(\varphi, \dot{\varphi}, w, \dot{w}): \Omega & \rightarrow \mathbb{R}^{3}, \\
\frac{\delta L}{\delta w}(\varphi, \dot{\varphi}, w, \dot{w}): \Omega & \rightarrow \mathbb{R}^{3}, \\
\frac{\delta^{\prime} L}{\delta w}(\varphi, \dot{\varphi}, w, \dot{w}): \partial \Omega & \rightarrow \mathbb{R}^{3}, \\
\frac{\delta L}{\delta \dot{w}}(\varphi, \dot{\varphi}, w, \dot{w}): \Omega & \rightarrow \mathbb{R}^{3} .
\end{aligned}
$$

Equation (2.5.1) then is equivalent to the system of differential equations

$$
\begin{aligned}
\frac{d \phi}{d t} & =\frac{\delta H}{\delta \dot{\phi}}(\phi, \dot{\phi}), \\
\frac{d \dot{\phi}}{d t} & =-\frac{\delta H}{\delta \phi}(\phi, \dot{\phi}),
\end{aligned}
$$


with boundary conditions

$$
\frac{\delta^{\prime} H}{\delta \phi}(\phi, \dot{\phi})=0 \text { on } \partial(\Omega \times[-\epsilon, \epsilon])
$$

Equation (2.5.2) is equivalent to the system of differential equations

$$
\begin{aligned}
\frac{d \varphi}{d t} & =\frac{1}{2 \epsilon} \frac{\delta\left(H \circ A_{\epsilon}\right)}{\delta \dot{\varphi}}(\varphi, \dot{\varphi}, w, \dot{w}), \\
\frac{d \dot{\varphi}}{d t} & =-\frac{1}{2 \epsilon} \frac{\delta\left(H \circ A_{\epsilon}\right)}{\delta \varphi}(\varphi, \dot{\varphi}, w, \dot{w}), \\
\frac{d w}{d t} & =\frac{3}{2 \epsilon^{3}} \frac{\delta\left(H \circ A_{\epsilon}\right)}{\delta \dot{w}}(\varphi, \dot{\varphi}, w, \dot{w}), \\
\frac{d \dot{w}}{d t} & =-\frac{3}{2 \epsilon^{3}} \frac{\delta\left(H \circ A_{\epsilon}\right)}{\delta w}(\varphi, \dot{\varphi}, w, \dot{w}),
\end{aligned}
$$

with boundary conditions

$$
\begin{aligned}
& \frac{\delta^{\prime}\left(H \circ A_{\epsilon}\right)}{\delta \varphi}(\varphi, \dot{\varphi}, w, \dot{w})=0, \\
& \frac{\delta^{\prime}\left(H \circ A_{\epsilon}\right)}{\delta w}(\varphi, \dot{\varphi}, w, \dot{w})=0 .
\end{aligned}
$$

Now let the specific $H$ be given as in the introduction:

$$
H=\iiint_{\Omega \times[-\epsilon, \epsilon]}\left(\frac{1}{2} \dot{\phi} \cdot \dot{\phi}+g\left(x, y, \phi, \phi_{x}, \phi_{y}, \epsilon\right)\right) d^{2} x d y .
$$

We can also consider the case of non-constant mass density, but since that case is similar, we assume the reference mass density is equal to 1 .

The equations (2.5.3) in this case are equivalent to

$$
\frac{d^{2} \phi}{d t^{2}}=-\frac{\partial g}{\partial \phi}+\operatorname{div} \frac{\partial g}{\partial(u, v)}
$$

where

$$
\frac{\partial g}{\partial(u, v)}:=\left(\begin{array}{ccc}
\frac{\partial g}{\partial u_{1}^{1}} & \frac{\partial g}{\partial u_{1}^{2}} & \frac{\partial g}{\partial v_{1}} \\
\frac{\partial g}{\partial u_{2}^{1}} & \frac{\partial g}{\partial u_{2}^{2}} & \frac{\partial g}{\partial v_{2}} \\
\frac{\partial g}{\partial u_{3}^{1}} & \frac{\partial g}{\partial u_{3}^{2}} & \frac{\partial g}{\partial v_{3}}
\end{array}\right)
$$

and where

$$
\begin{array}{ll}
u:=\partial_{x} \phi \quad u^{i}:=\partial_{x_{i}} \phi \quad u_{j}^{i}:=\partial_{x_{i}} \phi_{j} \\
v:=\partial_{y} \phi \quad v_{j}:=\partial_{y} \phi_{j} \quad i=1,2 ; j=1,2,3
\end{array}
$$


The divergence is taken row-wise in (2.5.8). The boundary condition (2.5.4) reads as

$$
\frac{\partial g}{\partial(u, v)} \cdot n=0 \text { on } \partial(\Omega \times[-\epsilon, \epsilon])
$$

where $n: \partial(\Omega \times[-\epsilon, \epsilon]) \rightarrow \mathbb{R}^{3}$ is the vector field of outer unit normal vectors to $\Omega \times[-\epsilon, \epsilon]$ (defined at the smooth points of the boundary). In the special case that the shell is periodic in the $x_{1}$ and $x_{2^{-}}$directions, the boundary condition (2.5.9) simplifies to

$$
\frac{\partial g}{\partial v}=0 \text { for } y= \pm \epsilon .
$$

The function $H \circ A_{\epsilon}: T \mathcal{M}_{2 d} \rightarrow \mathbb{R}$ has the form

$$
\begin{aligned}
\left(H \circ A_{\epsilon}\right)(\varphi, \dot{\varphi}, w, \dot{w}) & =\iint_{\Omega}\left(\epsilon \dot{\varphi}^{2}+\frac{\epsilon^{3}}{3} \dot{w}^{2}+2 \epsilon g\left(x, 0, \varphi, \varphi_{x}, w, \epsilon\right)\right. \\
+ & \left.\frac{\epsilon^{3}}{3}\left(g_{y, y}+2 g_{y, \phi} w+2 g_{y, u^{i}} w_{x_{i}}+g_{\phi, \phi} w^{2}+2 g_{\phi, u^{i}} w w_{x_{i}}+g_{u^{i}, u^{j}} w_{x_{i}} w_{x_{j}}\right)+O\left(\epsilon^{5}\right)\right) d^{2} x
\end{aligned}
$$

where, for example, $g_{\phi, \phi} w^{2}$ is shorthand for applying the bilinear form

$$
\frac{\partial^{2} g}{\partial \phi^{2}}\left(x, 0, \varphi, \varphi_{x}, w, \epsilon\right)
$$

to the pair of vectors $(w, w)$ and where the second derivatives under the integral sign have to be evaluated at $\left(x, 0, \varphi, \varphi_{x}, w, \dot{w}\right)$. We have

$$
\begin{aligned}
& \frac{\delta\left(H \circ A_{\epsilon}\right)}{\delta \varphi}=2 \epsilon g_{\phi}-2 \epsilon \operatorname{div}_{x} g_{u^{i}}+\frac{\epsilon^{3}}{3}\left(g_{y, y, \phi}+2 g_{y, \phi, \phi} w+2 g_{y, \phi, u^{i}} w_{x_{i}}\right. \\
& \left.+g_{\phi, \phi, \phi} w^{2}+2 g_{\phi, \phi, u^{i}} w w_{x_{i}}+g_{\phi, u^{i}, u^{j}} w_{x_{i}} w_{x_{j}}\right) \\
& -\frac{\epsilon^{3}}{3} \operatorname{div}\left(g_{y, y, u_{j}^{i}}+2 g_{y, \phi, u_{j}^{i}} w+2 g_{y, u^{k}, u_{j}^{i}} w_{x_{k}}+g_{\phi, \phi, u_{j}^{i}} w^{2}\right. \\
& \left.+2 g_{\phi, u^{k}, u_{j}^{i}} w_{x_{k}}+g_{u^{k}, u^{l}, u_{j}^{i}} w_{x_{k}} w_{x_{l}}\right)+O\left(\epsilon^{5}\right) \text {, } \\
& \frac{\delta^{\prime}\left(H \circ A_{\epsilon}\right)}{\delta \varphi}=2 \epsilon\left\langle g_{u}, n\right\rangle+\frac{\epsilon^{3}}{3}\left(g_{y, y, u_{j}^{i}}+2 g_{y, \phi, u_{j}^{i}} w+2 g_{y, u^{k}, u_{j}^{i}} w_{x_{k}}\right. \\
& \left.+g_{\phi, \phi, u_{j}^{i}} w^{2}+2 g_{\phi, u^{k}, u_{j}^{i}} w_{x_{k}}+g_{u^{k}, u^{l}, u_{j}^{i}} w_{x_{k}} w_{x_{l}}\right) \cdot n+O\left(\epsilon^{5}\right) \text {, } \\
& \frac{\delta\left(H \circ A_{\epsilon}\right)}{\delta w}=2 \epsilon g_{v}+\frac{\epsilon^{3}}{3}\left(g_{y, y, v}+2 g_{y, \phi}+2 g_{y, \phi, v} w+2 g_{y, u^{i}, v} w_{x_{i}}\right. \\
& -2\left(g_{y, u^{i}}\right)_{x_{i}}+2 g_{\phi, \phi} w+2 g_{\phi, u^{i}, v} w w_{x_{i}}+2 g_{\phi, u^{i}} w_{x_{i}} \\
& \left.-2\left(g_{\phi, u^{i}} w\right)_{x_{i}}+g_{u^{i}, u^{j}, v} w_{x_{i}} w_{x_{j}}-2\left(g_{u^{i}, u^{j}} w_{x_{i}}\right)_{x_{j}}\right)+O\left(\epsilon^{5}\right), \\
& \frac{\delta^{\prime}\left(H \circ A_{\epsilon}\right)}{\delta w}=\frac{2 \epsilon^{3}}{3}\left(\frac{\partial^{2} g}{\partial y \partial u}+\frac{\partial^{2} g}{\partial \phi \partial u} w+\frac{\partial^{2} g}{\partial u^{2}} \frac{\partial w}{\partial x}\right) \cdot n+O\left(\epsilon^{5}\right) \text {. }
\end{aligned}
$$

It is of interest to see how well the function $\varphi+y w$ satisfies the system of differential equations for 3d elasticity if $(\varphi, w)$ is a solution for the differential equation in the director-model approximation. Since the top and bottom faces are missing in the two dimensional model, it is especially interesting to see, how well the function $\varphi+y w$ satisfies the boundary conditions of our $3 \mathrm{~d}$ problem at the upper and the lower face of the plate. 


\subsection{A Simple Example: an Unconstrained Wave Equation}

We study the problem posed at the end of the preceding section for the following example. Choose $\Omega:=T^{2}$ and let in $(2.5 .7)$

$$
g=\frac{1}{2}\left(\phi_{x}^{2}+\phi_{y}^{2}-2 \phi \phi_{y}\right)
$$

The equations of motion (2.5.8) read

$$
\frac{d^{2} \phi}{d t^{2}}=\triangle_{x} \phi+\frac{\partial^{2} \phi}{\partial y^{2}}
$$

with boundary conditions

$$
\phi_{y}=\phi \text { for } y= \pm \epsilon .
$$

The equations of motion for the corresponding one-director model (2.5.5) are

$$
\begin{aligned}
\frac{d^{2} \varphi}{d t^{2}} & =\varphi_{x x}+w \\
\frac{d^{2} w}{d t^{2}} & =w_{x x}+\frac{3}{\epsilon^{2}}(w-\varphi) .
\end{aligned}
$$

Note the singular term in the equation (2.6.5).

To solve these differential equations, we make the Ansatz

$$
w:=c \cdot \varphi \text {. }
$$

Substitute this into the equations (2.6.4)-(2.6.5) to get the algebraic equation

$$
\frac{3}{\epsilon^{2}}(c-1)=c^{2}
$$

for the real number $c$. Now expand $c$ into a power series in $\epsilon$. We get

$$
c=1+\frac{1}{3} \epsilon^{2}+O\left(\epsilon^{3}\right) .
$$

Thus,

$$
w=\left(1+\frac{1}{3} \epsilon^{2}+O\left(\epsilon^{3}\right)\right) \cdot \varphi .
$$

We see that the boundary conditions (2.6.3) are satisfied to first order in $\epsilon$ by the function $\varphi+y w$

Now we return to the general case. From equations (2.5.5) and equations (2.5.11)-(2.5.14) we see that in the general case the equations for the director field are

$$
\frac{d^{2} w}{d t^{2}}=-\frac{3}{\epsilon^{2}} \frac{\partial g}{\partial v}+O(1)
$$

which contains a singular term of order $1 / \epsilon^{2}$. If

$$
\frac{\partial g}{\partial v}=O\left(\epsilon^{2}\right),
$$

then the singularity in (2.6.10) disappears. We are led to an unconstrained Cosserat-model. In this situation the material is anisotropic and mechanical properties depend on its thickness. 


\subsection{Unconstrained Limiting Models}

Here is one way the situation described in the last subsection arises. We start with a specific isotropic 3d elasticity model (Saint Venant-Kirchhoff, for instance) that has a energy density function of the form

$$
\frac{1}{2} \dot{\phi} \cdot \dot{\phi}+g_{\text {isotropic }}\left(x, y, \phi, \frac{\partial \phi}{\partial x}, \frac{\partial \phi}{\partial y}\right)
$$

Then consider a scaled Hamiltonian

$$
\begin{aligned}
H & =\iint_{\Omega} \int_{-\epsilon}^{\epsilon}\left(\frac{1}{2} \dot{\phi} \cdot \dot{\phi}+g\left(x, y, \phi, \frac{\partial \phi}{\partial x}, \frac{\partial \phi}{\partial y}, \epsilon\right)\right) d^{2} x d y \\
& :=\iint_{\Omega} \int_{-\epsilon}^{\epsilon}\left(\frac{1}{2} \dot{\phi} \cdot \dot{\phi}+g_{\text {isotropic }}\left(x, y, \phi, \frac{\partial \phi}{\partial x}, \epsilon \frac{\partial \phi}{\partial y}\right)\right) d^{2} x d y .
\end{aligned}
$$

In particular, if $g$ is quadratic plus higher order terms in $\partial \phi / \partial y$, then it satisfies the hypotheses needed to get an unconstrained limiting model. The Hamiltonian for the limiting model then is given in terms of the the variable $\varphi$ and the scaled variable $\bar{w}=\epsilon w$ by

$$
H_{\text {shell }}=\iint_{\Omega}\left(\frac{1}{2}\left(\dot{\varphi} \cdot \dot{\varphi}+\frac{1}{3} \dot{\bar{w}} \cdot \dot{\bar{w}}\right)+g_{\text {shell }}\left(x, \varphi, \bar{w}, \frac{\partial \varphi}{\partial x}\right)\right) d^{2} x
$$

where

$$
g_{\text {shell }}\left(x, \varphi, \bar{w}, \frac{\partial \varphi}{\partial x}\right)=g_{\text {isotropic }}\left(x, 0, \varphi, \frac{\partial \varphi}{\partial x}, \bar{w}\right)
$$

Alternatively we can write

$$
H_{\text {shell }}=\lim _{\epsilon \rightarrow 0} \frac{1}{2 \epsilon}\left(H \circ A_{\epsilon}\right)\left(\varphi, \frac{\bar{w}}{\epsilon}, \dot{\varphi}, \frac{\dot{\bar{w}}}{\epsilon}\right) .
$$

Then the limiting system has a single unconstrained director.

Now we look at the convergence of the $3 \mathrm{~d}$ Poisson bracket. Introduce

$$
\left\{F_{1}, F_{2}\right\}_{\text {shell }}=2 \epsilon\left\{F_{1}, F_{2}\right\}_{2 d}^{\epsilon}=D_{\varphi} F_{1} \frac{\delta F_{2}}{\delta \dot{\varphi}}+3 D_{\bar{w}} F_{1} \frac{\delta F_{2}}{\delta \dot{\bar{w}}}-D_{\varphi} F_{2} \frac{\delta F_{1}}{\delta \dot{\varphi}}-3 D_{\bar{w}} F_{2} \frac{\delta F_{1}}{\delta \dot{\bar{w}}} .
$$

Corollary 2.3 For functions $F_{1}, F_{2}$, satisfying (2.6.11), one has

$$
\left\{F_{1, \text { shell }}, F_{2, \text { shell }}\right\}_{\text {shell }}(\varphi, \bar{w}, \dot{\varphi}, \dot{\bar{w}})=\lim _{\epsilon \rightarrow 0} \frac{1}{2 \epsilon}\left(\left\{F_{1}, F_{2}\right\}_{3 d}^{\epsilon} \circ A_{\epsilon}\right)\left(\varphi, \frac{\bar{w}}{\epsilon}, \dot{\varphi}, \frac{\dot{\bar{w}}}{\epsilon}\right) .
$$

In the next section we deal with the situation that condition (2.6.11) is not satisfied. As we shall see, this leads to a special kind of one-director model in which the director field is not independent from the displacement of the shell, instead, it is a function of the displacement and its derivatives. For lack of terminology, we shall call this model as a constrained-director model. 


\section{Constrained-director Shell Models}

We now consider the case when the limiting procedures lead to constraints in the limiting shell model. Such constraints need to be distinguished from any additional constraints one may wish to impose. In this section we consider the simplest examples, and leave the example of the Kirchhoff shell for the next section. We note that constraints can involve derivatives and this naturally leads one from second order elasticity models to higher order ones. We shall see this explicitly in the Kirchhoff rod and shell models.

\subsection{The Limit of the Hamiltonian Structure}

Consider a 3d elastic body with Hamiltonian of the form

$$
H=\iint_{\Omega} \int_{-\epsilon}^{\epsilon}\left(\frac{1}{2} \dot{\phi} \cdot \dot{\phi}+g\left(x, y, \phi, \partial_{x} \phi, \partial_{y} \phi, \epsilon\right)\right) d^{2} x d y
$$

We assume that the boundary condition in the $x$-direction is periodic for simplicity. The elastodynamical equation is

$$
\frac{d^{2} \phi}{d t^{2}}=-\frac{\partial g}{\partial \phi}+\operatorname{div} \frac{\partial g}{\partial(u, v)} \quad \text { where } \quad v=\partial_{y} \phi
$$

with the boundary conditions

$$
\frac{\partial g}{\partial v}=0, \quad \text { where } y= \pm \epsilon
$$

Throughout this section we assume that equation (2.6.11) is not satisfied and that

Assumption A. $\quad \partial^{2} g / \partial v^{2}$ does not vanish anywhere.

As before, we take the approximation

$$
\phi(x, y) \approx \varphi(x)+y w(x) .
$$

However, here we require that the above approximation satisfies the boundary condition (3.1.2) up to order $\epsilon$. Under the Assumption A, this implies that $w$ can be written as $w(x)=\mathcal{L}\left(x, \varphi, \partial_{x} \phi\right)+$ $O(y)$ approximately, for some function $\mathcal{L}$. Thus, we define

$$
w(x):=\mathcal{L}\left(x, \varphi, \partial_{x} \varphi\right)
$$

That is, as mentioned before, the director is determined by $\varphi, \partial_{x} \varphi$. Note that the configuration space of the constrained-director model is

$$
\mathcal{M}_{c d}=\left\{\varphi \mid \varphi: T^{2} \rightarrow \mathbb{R}^{3} \text { is an embedding }\right\} .
$$

The Poisson bracket on $T \mathcal{M}_{c d}$ is

$$
\left\{F_{1}, F_{2}\right\}_{c d}=\iint_{\Omega}\left(\frac{\delta F_{1}}{\delta \varphi} \frac{\delta F_{2}}{\delta \dot{\varphi}}-\frac{\delta F_{2}}{\delta \varphi} \frac{\delta F_{1}}{\delta \dot{\varphi}}\right) d^{2} x
$$


At first glance, the 3d Poisson bracket will not converge to that of the constrained-director model. Nevertheless, as we shall see, for Hamiltonians of interests to us, such as those of the form of kinetic energy plus a potential energy, the 3d Poisson brackets do converge to that of the constrained-director model.

Introduce the embedding

$$
\mathcal{U}_{\epsilon}: \mathcal{M}_{c d} \rightarrow \mathcal{M}_{3 d}^{\epsilon}, \quad\left(\mathcal{U}_{\epsilon}\right)(x, y)=\varphi(x)+y \mathcal{L}(x, \varphi, \partial \varphi)
$$

Let $\mathcal{C}_{\epsilon}=T \mathcal{U}_{\epsilon}$ be the tangent map of $\mathcal{U}_{\epsilon}$. As we shall see, this is an almost-Poisson embedding for Hamiltonians of most interests:

Theorem 3.1 As $\epsilon \rightarrow 0$,

$$
\left\{F_{1}, F_{2}\right\}_{3 d}^{\epsilon} \circ \mathcal{C}_{\epsilon}=\frac{1}{2 \epsilon}\left\{F_{1} \circ \mathcal{C}_{\epsilon}, F_{2} \circ \mathcal{C}_{\epsilon}\right\}_{c d}+O\left(\epsilon^{2}\right)
$$

if either

1. both $F_{1}, F_{2}$ are of form (3.1.1), i.e., "kinetic energy plus potential," where the kinetic energy is the Riemannian metric,

2. $F_{1}$ is of the form of

$$
H=\iint_{\Omega} \int_{-\epsilon}^{\epsilon} m(x, y)\left(\frac{1}{2} \dot{\phi} \cdot \dot{\phi}+g\left(x, y, \phi, \partial_{x} \phi, \partial_{y} \phi, \epsilon\right)\right) d^{2} x d y,
$$

where $m$ is the mass density, and $g$ satisfies

$$
\frac{\partial g\left(x, y, \varphi+y \mathcal{L}, \partial_{x}(\varphi+y \mathcal{L}), \mathcal{L}\right)}{\partial v}=O(\epsilon)
$$

at $y= \pm \epsilon$ and where $F_{2}$ is arbitrary.

Proof We only need to prove

$$
\iint_{\Omega} \int_{-\epsilon}^{\epsilon}\left(\frac{\delta F_{1}}{\delta v} \cdot \partial_{y}\left(\frac{\delta F_{2}}{\delta \dot{\phi}}\right)\right) \circ \mathcal{C}_{\epsilon} d^{2} x d y=2 \epsilon \iint_{\Omega}\left(\frac{\delta F_{1}}{\delta v} \frac{\delta \mathcal{L}}{\delta \varphi} \frac{\delta F_{2}}{\delta \dot{\phi}}\right) \circ \mathcal{C}_{\epsilon} d^{2} x+O\left(\epsilon^{3}\right)
$$

where $v=\partial \phi / \partial y=\mathcal{L}$. We first prove this for the case 1 . In this case, one has

$$
\left(\partial_{y} \frac{\delta F_{i}}{\delta \dot{\phi}}\right) \circ \mathcal{C}_{\epsilon}=\left(\partial_{y}(\dot{\phi})\right) \circ \mathcal{C}_{\epsilon}=\dot{\mathcal{L}}
$$

On the other hand, since

$$
\frac{\delta F_{2}}{\delta \dot{\phi}} \circ \mathcal{C}_{\epsilon}=\dot{\phi}, \quad \frac{\delta \mathcal{L}}{\delta \varphi}=\partial_{\varphi} \mathcal{L}
$$

one has

$$
\frac{\delta F_{2}}{\delta \dot{\phi}} \frac{\delta v}{\delta \varphi}=\partial_{\varphi} \mathcal{L} \cdot \dot{\varphi}=\dot{\mathcal{L}}
$$

Now (3.1.4) follows from (3.1.5), (3.1.6). This proves the theorem in the case 1 .

For the proof of the case 2 , one simply notices that, in addition to the above relations, the compatibility condition (3.1.3) implies that both sides of (3.1.4) are of order $O\left(\epsilon^{3}\right)$. 


\section{Remark}

Note that $\phi(x, y)=\varphi(x)+y \mathcal{L}$ only satisfies the boundary conditions up to order $\epsilon$. Put in another way, it only satisfies

$$
\frac{\partial g}{\partial v}=O(\epsilon)
$$

which is much weaker than the condition (1.0.3). In order to satisfy the boundary conditions (3.1.2) exactly, or, to satisfy the condition (1.0.3), it is necessary to use a two-directors model,

$$
\phi(x, y) \approx \varphi(x)+y \mathcal{L}_{1}+y^{2} \mathcal{L}_{2}
$$

Thus the constrained-one-director model is only a first order approximation. Nevertheless, the Hamiltonian of the constrained-one-director model, $\mathcal{C}_{\epsilon}{ }^{*} E$, differs from that of the constrained-twodirectors model by a term of order $O\left(\epsilon^{2}\right)$. Thus, if one assumes that the boundary condition in the $x$-direction is periodic, the constrained-one-director model is good enough. However, if the boundary condition in the $x$-direction is not periodic, presumably a constrained-two directors model is better for the purpose of approximation, as a boundary layer might develop. But, as this layer is believed to be very thin, one expects that the constrained-director model still provides a good approximation in the interior (see also the remark in John [1971]).

Corollary 3.2 For functions $F_{1}, F_{2}$ as in the preceding theorem, one has

$$
\lim _{\epsilon \rightarrow 0} \frac{1}{2 \epsilon}\left(\left\{F_{1}, F_{2}\right\}_{3 d}^{\epsilon} \circ \mathcal{C}_{\epsilon}\right)=\left\{\lim _{\epsilon \rightarrow 0} \frac{1}{2 \epsilon} F_{1} \circ \mathcal{C}_{\epsilon}, \lim _{\epsilon \rightarrow 0} \frac{1}{2 \epsilon} F_{2} \circ \mathcal{C}_{\epsilon}\right\}_{\mathrm{cd}} .
$$

Now we show that the constrained-director model is compatible with the one-director model. Define

$$
\mathcal{U}_{1}: \mathcal{M}_{\mathrm{cd}} \rightarrow \mathcal{M}_{2 d} \quad \mathcal{U}_{1}: \varphi \rightarrow\left(\varphi, \mathcal{L}\left(x, \varphi, \partial_{x} \varphi\right)\right)
$$

and set $\mathcal{B}=T \mathcal{U}_{1}: T \mathcal{M}_{\mathrm{cd}} \rightarrow T \mathcal{M}_{2 d}$. Then $\mathcal{C}_{\epsilon}$ can be decomposed as $A_{\epsilon} \circ \mathcal{B} ;$ moreover, $\mathcal{B}$ is also an almost-Poisson embedding:

Corollary 3.3 Let $F_{1}, F_{2}$ be as in Theorem 3.1, then

$$
\left\{A_{\epsilon}^{*} F_{1}, A_{\epsilon}^{*} F_{2}\right\}_{2 d}^{\epsilon} \circ \mathcal{B}=\left\{\left(\mathcal{C}_{\epsilon}^{*} F_{1}\right),\left(\mathcal{C}_{\epsilon}^{*} F_{2}\right)\right\}_{\mathrm{cd}}+O\left(\epsilon^{3}\right) .
$$

\subsection{The Wave Equation as a Constrained Director Model}

In general, when the limiting model for a shell has constraints, these constraints must be worked out in each case and the constraint will depend on the constitutive function chosen. We illustrate the procedure first with some simplified examples to illustrate the ideas and then we consider the case of an inextensible Kirchhoff shell.

\section{Example 1.}

$$
\begin{aligned}
& \frac{\partial^{2} \phi}{\partial^{2} t}=\frac{\partial^{2} \phi}{\partial x_{1}^{2}}+\frac{\partial^{2} \phi}{\partial x_{2}^{2}}+\frac{\partial^{2} \phi}{\partial y^{2}}, \quad x=\left(x_{1}, x_{2}\right) \in T^{2},-\epsilon \leq y \leq \epsilon \\
& \partial_{y} \phi=0, \quad \text { for } y= \pm \epsilon
\end{aligned}
$$


The director is given by $w=0$. The limit equation is

$$
\partial_{t}^{2} \varphi=\left(\partial_{x_{1}}^{2}+\partial_{x_{2}}^{2}\right) \varphi, \quad x \in S^{1}
$$

This can also be seen from the fact that (3.2.1)-(3.2.2) has a family of special exact solutions:

$$
\phi(x, y)=\bar{\varphi}(x) \exp (y)
$$

where $\bar{\varphi}$ satisfies eq. (3.2.3).

Example 2. Consider the linear wave equation

$$
\frac{\partial^{2} \phi}{\partial^{2} t}=\frac{\partial^{2} \phi}{\partial x_{1}^{2}}+\frac{\partial^{2} \phi}{\partial x_{2}^{2}}+\frac{\partial^{2} \phi}{\partial y^{2}}, \quad x=\left(x_{1}, x_{2}\right) \in T^{2},-\epsilon \leq y \leq \epsilon
$$

with the boundary conditions

$$
\partial_{y} \phi=\phi, \quad \text { for } y= \pm \epsilon
$$

which has the Hamiltonian

$$
H(\phi, \dot{\phi})=\int_{S^{1}} \int_{-\epsilon}^{\epsilon} \frac{1}{2}\left(\left(\partial_{t} \phi\right)^{2}+\left(\partial_{x} \phi\right)^{2}+\left(\partial_{y} \phi\right)^{2}-2 \phi \phi_{y}\right) d^{2} x d y
$$

The director is given by $\mathcal{L}=\varphi$. Substitute

$$
\phi \approx(1+y) \varphi
$$

into equation (3.2.6) to obtain the Hamiltonian (modulo a multiplication by a constant) for the constrained-director model

$$
H_{\mathrm{cd}}=\int_{T^{2}} \frac{1}{2}\left(\left(\partial_{t} \varphi\right)^{2}+\left(\partial_{x} \varphi\right)^{2}-\varphi^{2}\right) d^{2} x
$$

The corresponding Hamiltonian system is

$$
\partial_{t}^{2} \varphi=\left(\partial_{x_{1}}^{2}+\partial_{x_{2}}^{2}\right) \varphi+\varphi, \quad x \in T^{2}
$$

This is the correct limit equation, as the original equation (3.2.4)-(3.2.5) has a family of special exact solutions:

$$
\phi(x, y)=\bar{\varphi}(x) \exp (y)
$$

where $\bar{\varphi}$ satisfies eq. (3.2.7).

Example 3. Consider the following system of hyperbolic equations in linear elasticity on the domain $T^{2} \times[-\epsilon, \epsilon]$ with points denoted by $\left(x_{1}, x_{2}, y\right)$,

$$
\begin{aligned}
& \partial_{t}^{2} \phi_{i}=\partial_{j}\left\{\lambda e_{p p}(\vec{\phi}) \delta_{i j}+2 \mu e_{i j}(\vec{\phi})\right\} \\
& \lambda e_{p p}(\vec{\phi}) \delta_{i 3}+2 \mu e_{i 3}(\vec{\phi})=0, \quad \text { for } y= \pm \epsilon, \quad i=1,2,3 \text {, }
\end{aligned}
$$


where $\vec{\phi}=\left(\phi_{1}, \phi_{2}, \phi_{3}\right), e_{i j}(\vec{\phi})=\frac{1}{2}\left(\partial_{i} \phi_{j}+\partial_{j} \phi_{i}\right)$ is the linearized strain tensor, $\lambda, \mu$ the Lamé constants. Here we used $\partial_{1}:=\frac{\partial}{\partial x_{1}}, \partial_{2}:=\frac{\partial}{\partial x_{2}}$ and $\partial_{3}:=\frac{\partial}{\partial y}$. Note that these equations describe a linearized Saint Venant-Kirchhoff model.

The Hamiltonian of this system is

$$
\iiint_{T^{2} \times[-\epsilon, \epsilon]}\left\{\partial_{t} \vec{\phi} \cdot \partial_{t} \vec{\phi}+\lambda e_{p p}(\vec{\phi}) e_{q q}(\vec{\phi})+2 \mu\left(e_{i j}(\vec{\phi})\right)^{2}\right\} d^{2} x d y
$$

The equations considered in Ciarlet and Miara [1992] correspond to (and are more general than) the stationary case of equations (3.2.8), (3.2.9).

As in previous examples, we take an approximation by a constrained-director model

$$
\vec{\phi} \approx \vec{\varphi}+y \vec{w}
$$

where $\vec{\varphi}=\left(\varphi_{1}, \varphi_{2}, \varphi_{3}\right), \vec{w}$ are vector-valued functions on $\mathbb{R}^{3}$. The director is obtained from (3.2.9)

$$
\vec{w}=\left(-\partial_{1} \varphi_{3},-\partial_{2} \varphi_{3},-\frac{\lambda}{2 \mu+\lambda}\left(\partial_{1} \varphi_{1}+\partial_{2} \varphi_{2}\right)\right) .
$$

The corresponding $e_{i j}$ 's are

$$
\begin{aligned}
e_{11} & =\partial_{1} \varphi_{1}-y \partial_{11}^{2} \varphi_{3}, \\
e_{12} & =\frac{1}{2}\left(\partial_{2} \varphi_{1}+\partial_{1} \varphi_{2}-2 y \partial_{12}^{2} \varphi_{3}\right), \\
e_{22} & =\partial_{2} \varphi_{2}-y \partial_{22}^{2} \varphi_{3} \\
e_{13} & =-\frac{\lambda}{2(2 \mu+\lambda)}\left(\partial_{11}^{2} \varphi_{1}+\partial_{12}^{2} \varphi_{2}\right) y, \\
e_{23} & =-\frac{\lambda}{2(2 \mu+\lambda)}\left(\partial_{12}^{2} \varphi_{1}+\partial_{22}^{2} \varphi_{2}\right) y, \\
e_{33} & =-\frac{\lambda}{2 \mu+\lambda}\left(\partial_{1} \varphi_{1}+\partial_{2} \varphi_{2}\right) .
\end{aligned}
$$

Thus, the corresponding Hamiltonian is

$$
2 \epsilon \iint_{T^{2}} \partial_{t} \vec{\varphi} \cdot \partial_{t} \vec{\varphi} d^{2} x
$$

plus the potential energy

$$
\begin{aligned}
& \iint_{T^{2}} \int_{-\epsilon}^{\epsilon}\left\{\lambda\left(\partial_{1} \varphi_{1}-y \partial_{11}^{2} \varphi_{3}+\partial_{2} \varphi_{2}-y \partial_{22}^{2} \varphi_{3}-\frac{\lambda}{2 \mu+\lambda}\left(\partial_{1} \varphi_{1}+\partial_{2} \varphi_{2}\right)\right)^{2}++2 \mu\left(\left(\partial_{1} \varphi_{1}-y \partial_{11}^{2} \varphi_{3}\right)^{2}\right.\right. \\
& \left.\left.+\frac{1}{2}\left(\partial_{2} \varphi_{1}+\partial_{1} \varphi_{2}-2 y \partial_{12}^{2} \varphi_{3}\right)^{2}+\left(\partial_{2} \varphi_{2}-y \partial_{22}^{2} \varphi_{3}\right)^{2}+\left(\frac{\lambda}{2 \mu+\lambda}\left(\partial_{1} \varphi_{1}+\partial_{2} \varphi_{2}\right)\right)^{2}\right)\right\} d^{2} x d y+O\left(\epsilon^{3}\right) \\
= & 2 \epsilon \iint_{T^{2}}\left\{\frac{4 \lambda \mu^{2}}{(\lambda+2 \mu)^{2}}\left(\partial_{1} \varphi_{1}+\partial_{2} \varphi_{2}\right)^{2}+2 \mu\left(\left(\partial_{1} \varphi_{1}\right)^{2}+\frac{1}{2}\left(\partial_{2} \varphi_{1}+\partial_{1} \varphi_{2}\right)^{2}+\left(\partial_{2} \varphi_{2}\right)^{2}\right) d^{2} x+0\left(\epsilon^{3}\right) .\right.
\end{aligned}
$$

[1970]). Since the boundary condition in the $x$-direction is periodic, the higher order term can be ignored, so the limit Hamiltonian is 


$$
\begin{aligned}
& \lim _{\epsilon \rightarrow 0} \frac{1}{2 \epsilon}\left(H \circ A_{\epsilon}\right) \\
&\left.\quad=\iint_{T^{2}}\left\{\sum_{i=1}^{2} \partial_{t} \varphi_{i} \cdot \partial_{t} \varphi_{i}+\frac{2 \mu \lambda}{\lambda+2 \mu}\left(\partial_{1} \varphi_{1}+\partial_{2} \varphi_{2}\right)^{2}+2 \mu\left(\partial_{1} \varphi_{1}\right)^{2}+\mu\left(\partial_{2} \varphi_{1}+\partial_{1} \varphi_{2}\right)^{2}+2 \mu\left(\partial_{2} \varphi_{2}\right)^{2}\right)\right\} d^{2} x \\
& \quad=\iint_{T^{2}}\left\{\sum_{i=1}^{2} \partial_{t} \varphi_{i} \cdot \partial_{t} \varphi_{i}+\frac{2 \mu \lambda}{\lambda+2 \mu} e_{p p}(\vec{\varphi}) e_{q q}(\vec{\varphi})+2 \mu\left(e_{i j}(\vec{\varphi})\right)^{2}\right\} d^{2} x
\end{aligned}
$$

The limiting $2 \mathrm{~d}$ equation is

$$
\partial_{t}^{2} \varphi_{i}=\partial_{j}\left\{\frac{2 \lambda \mu}{\lambda+2 \mu} e_{p p}(\vec{\varphi}) \delta_{i j}+2 \mu e_{i j}(\vec{\varphi})\right\}, \quad i=1,2
$$

which corresponds to a membrane model.

\section{The Kirchhoff Shell as a Limit of a 3d Saint Venant-Kirchhoff Material}

\subsection{Saint Venant-Kirchhoff Materials}

Material frame invariance implies that the stored energy density of a hyperelastic material is of the form

$$
g(x, y, \phi, D \phi)=W\left(D \phi^{T} D \phi\right)
$$

(compare Marsden and Hughes [1994], Ch. 3). Let

$$
E:=\frac{1}{2}\left(D \phi^{T} D \phi-1\right)
$$

denote the Lagrangian strain tensor. A Saint Venant-Kirchhoff material is defined by its constitutive equations

$$
S=\lambda \operatorname{tr}(E) \operatorname{Id}+2 \mu E,
$$

where $S$ is the stress tensor and the numbers $\lambda$ and $\mu$ are the Lamé constants (compare Fox, Raoult and Simo [1993]). Saint Venant-Kirchhoff materials as defined above are actually hyperelastic with stored energy density given by

$$
W\left(D \phi^{T} D \phi\right)=\frac{\mu}{4}\left(\sum_{i, j=1}^{3}\left(z_{i} \cdot z_{j}-\delta_{i j}\right)^{2}\right)+\frac{\lambda}{8}\left(\sum_{i=1}^{3}\left(\left\|z_{i}\right\|^{2}-1\right)\right)^{2},
$$

where $z_{i}:=\partial \phi / \partial x_{i}, i=1,2,3$ and $x_{1}, x_{2}, x_{3}$ are Cartesian coordinates in $\mathbb{R}^{3}$. To see this, one has to check that

$$
S=2 \nabla W\left(D \phi^{T} D \phi\right),
$$

which is a straightforward computation (cf. Marsden and Hughes [1994]). Using the Saint VenantKirchhoff constitutive equations in modeling a given elastic material can be interpreted as using a truncation of the real constitutive equations of this material (cf. Fox, Raoult and Simo [1993]). 


\subsection{The Kirchhoff Membrane Model}

In this section we apply our general methods to derive constrained-director models outlined above to Saint Venant-Kirchhoff materials.

Following our general procedure we rewrite the coordinates in $\mathbb{R}^{3}$ as $x_{1}, x_{2}, y$ and use the notation $u^{i}=\partial \phi / \partial x_{i}, i=1,2$ and $v=\partial \phi / \partial y$. We make the Ansatz

$$
\phi(x, y)=\varphi(x)+y w(x)
$$

for the configuration of the $3 \mathrm{~d}$ body and determine the director field $w$ from the condition

$$
\frac{\partial g}{\partial v}(\varphi+y w)=O(\epsilon)
$$

This equation is satisfied if

$$
\begin{aligned}
& u^{1} \cdot w=O(\epsilon), \\
& u^{2} \cdot w=O(\epsilon),
\end{aligned}
$$

and if

$$
(2 \mu+\lambda)\left(\|w\|^{2}-1\right)+\lambda\left(\sum_{\alpha=1}^{2}\left\|u^{\alpha}\right\|^{2}-1\right)=O(\epsilon) .
$$

After solving for $w$ in terms of $\partial_{x_{1}} \varphi$ and $\partial_{x_{2}} \varphi$, inserting the result into $W$, and dropping higher order terms, we arrive at a first-order shell model with potential energy density

$$
\begin{aligned}
W_{m}\left(s_{1}, s_{2}\right) & =\frac{\mu}{4}\left(\sum_{\alpha, \beta=1}^{2}\left(s_{\alpha} \cdot s_{\beta}-\delta_{\alpha, \beta}\right)^{2}\right)+\frac{\lambda}{8}\left(\sum_{\alpha=1}^{2}\left(\left\|s_{\alpha}\right\|^{2}-1\right)\right)^{2}-\frac{\lambda^{2}}{8(2 \mu+\lambda)}\left(\sum_{\alpha=1}^{2}\left(\left\|s_{\alpha}\right\|^{2}-1\right)\right)^{2} \\
& =\frac{\mu}{4}\left(\sum_{\alpha, \beta=1}^{2}\left(s_{\alpha} \cdot s_{\beta}-\delta_{\alpha, \beta}\right)^{2}\right)+\frac{2 \mu \lambda}{8(2 \mu+\lambda)}\left(\sum_{\alpha=1}^{2}\left(\left\|s_{\alpha}\right\|^{2}-1\right)\right)^{2}
\end{aligned}
$$

where $s_{\alpha}=\partial_{x_{\alpha}} \varphi$ for $i=1,2$. Note that $W_{m} \geq 0$ and $W_{m}=0$ if and only if

$$
\begin{aligned}
s_{1} \cdot s_{2} & =0 \\
\left\|s_{1}\right\|^{2}-1 & =0 \\
\left\|s_{2}\right\|^{2}-1 & =0 .
\end{aligned}
$$

We see that the application of our asymptotic procedure to a 3d Saint Venant-Kirchhoff material leads to an $2 \mathrm{~d}$ membrane model that does not contain any director field.

\subsection{The Inextensible Kirchhoff Shell}

In the last subsection we derived a $2 \mathrm{~d}$ membrane model with potential energy $W_{m}$. Now consider the equilibrium problem. Assume that the equilibrium state has a limit as $\epsilon \rightarrow 0$, then the limit equilibrium has potential energy either $W_{m}>0$ or $W_{m}=0$. In the former case it is determined by the Euler-Lagrangian equation and the boundary conditions, so we can stop here. However, if 
$W_{m}=0$, then the Euler-Lagrangian equation and the boundary conditions alone do not determine the limit equilibrium, and we need to consider a higher order approximation, which will be the aim of this subsection.

Again, we write

$$
\phi\left(x_{1}, x_{2}, y\right)=\varphi\left(x_{1}, x_{2}\right)+w\left(x_{1}, x_{2}\right) y,
$$

where $w$ is the director field. But now we introduce the inextensibility constraint that $\partial \varphi / \partial x_{1}$ and $\partial \varphi / \partial x_{2}$ are orthonormal vectors, which is equivalent to setting $W_{m}=0$. The preceding discussion shows that one has to choose the director field $w$ so that

$$
\frac{\partial \varphi}{\partial x_{1}}, \frac{\partial \varphi}{\partial x_{2}} \text { and } w
$$

are orthonormal. This means that $w$ is a unit normal vector field to the surface which is parametrized by the map $\varphi$. Asking that

$$
\operatorname{det}\left(\frac{\partial \varphi}{\partial x_{1}}, \frac{\partial \varphi}{\partial x_{2}}, w\right)>0
$$

uniquely determines the director field $w$. Obviously, one has

$$
w=\frac{\partial \varphi}{\partial x_{1}} \times \frac{\partial \varphi}{\partial x_{2}}
$$

Substituting the Ansatz $\phi=\varphi+y w$ into the Saint Venant-Kirchhoff potential energy density and dropping higher order terms in $\epsilon$ we get the potential energy density for an inextensible shell, given by

$$
W_{s}\left(\frac{\partial \varphi}{\partial x_{\alpha}}, \frac{\partial w}{\partial x_{\alpha}}\right)=\frac{\mu}{4} \sum_{\alpha, \beta=1}^{2}\left(\frac{\partial \varphi}{\partial x_{\alpha}} \cdot \frac{\partial w}{\partial x_{\beta}}+\frac{\partial \varphi}{\partial x_{\beta}} \cdot \frac{\partial w}{\partial x_{\alpha}}\right)^{2}+\frac{\lambda}{2}\left(\sum_{\alpha=1}^{2} \frac{\partial \varphi}{\partial x_{\alpha}} \cdot \frac{\partial w}{\partial x_{\alpha}}\right)^{2}
$$

which is the potential energy for the inextensional shell model.

We want to give an interpretation of this energy density in terms of geometric data of the surface $\Sigma$ parametrized by the map $\varphi$. Remember that the first fundamental form

$$
E\left(d x_{1}\right)^{2}+2 F d x_{1} d x_{2}+G\left(d x_{2}\right)^{2}
$$

of the surface $\Sigma$ is defined by

$$
\begin{aligned}
E & :=\frac{\partial \varphi}{\partial x_{1}} \cdot \frac{\partial \varphi}{\partial x_{1}} \\
F & :=\frac{\partial \varphi}{\partial x_{1}} \cdot \frac{\partial \varphi}{\partial x_{2}} \\
G & :=\frac{\partial \varphi}{\partial x_{2}} \cdot \frac{\partial \varphi}{\partial x_{2}}
\end{aligned}
$$

and that its second fundamental form

$$
e\left(d x_{1}\right)^{2}+2 f d x_{1} d x_{2}+g\left(d x_{2}\right)^{2}
$$


is defined by

$$
\begin{aligned}
e & :=-\frac{\partial \varphi}{\partial x_{1}} \cdot \frac{\partial n}{\partial x_{1}} \\
f & :=-\frac{\partial \varphi}{\partial x_{1}} \cdot \frac{\partial n}{\partial x_{2}} \\
& =-\frac{\partial \varphi}{\partial x_{2}} \cdot \frac{\partial n}{\partial x_{1}} \\
g & :=-\frac{\partial \varphi}{\partial x_{2}} \cdot \frac{\partial n}{\partial x_{2}}
\end{aligned}
$$

Alternatively, the second fundamental form $\Pi_{p}$ at a point $p \in \Sigma$ is the quadratic form associated to (4.3.2) which takes the value $\Pi_{p}(v)=e v_{1}^{2}+2 f v_{1} v_{2}+g v_{2}^{2}$ at $v=v_{1} \partial \varphi / \partial x_{1}+v_{2} \partial \varphi / \partial x_{2} \in T_{p} \Sigma$. The mean curvature $H$ of the surface $\Sigma$, which is half the sum of the principle curvatures $k_{1}, k_{2}$ at every point of the surface, can then be expressed as

$$
H=\frac{1}{2} \frac{e G-2 f F+g E}{E G-F^{2}} .
$$

The Gaussian curvature $K=k_{1} k_{2}$ can be written as

$$
K=\frac{e g-f^{2}}{E G-F^{2}} .
$$

By choice of the map $\varphi$, the first fundamental form is just the identity matrix and the formulas for the mean curvature and the Gaussian curvature reduce to

$$
H=\frac{1}{2}(e+g),
$$

and

$$
K=e g-f^{2} .
$$

We can therefore rewrite the stored energy density of the inextensible shell as

$$
W_{s}(H, K)=(4 \mu+2 \lambda) H^{2}-2 \mu K \text {. }
$$

We see that under the additional assumption of inextensibility our method leads to an inextensible $2 \mathrm{~d}$ shell model without any director variables, the energy density of which can be expressed in terms of mean and Gaussian curvature only. This is in sharp contrast to the membrane model derived earlier where the energy density depends only on the first fundamental form.

\section{The Convergence of 3d Elasticity to Rod Models}

\subsection{Limits of Hamiltonian Structures}

The methods in the previous sections also apply to thin rods. For a general introduction to the theory of rods, see Antman [1972], and for the Hamiltonian formulation of rod theories see Simo, 
Marsden and Krishnaprasad [1988] and references therein. We indicate in this section how to carry out this program.

Consider the motion of a rod with reference configuration given by

$$
R_{\epsilon}=\left\{(x, y, z) \mid x^{2}+y^{2} \leq \epsilon^{2}, 0 \leq z \leq l\right\} \subset \mathbb{R}^{3}
$$

which consists of embeddings

$$
\phi: R_{\epsilon} \rightarrow \mathbb{R}^{3}
$$

Expanding $\phi$ at $x=y=0$, we obtain

$$
\varphi(z)=\phi(0,0, z), w_{1}(z)=\frac{\partial \phi}{\partial x}(0,0, z), w_{2}(z)=\frac{\partial \phi}{\partial y}(0,0, z),
$$

where $w_{1}, w_{2}$ are the director fields.

We introduce an approximation embedding $\mathcal{K}_{\epsilon}$ as the tangent lift of the map

$$
\left(\varphi(z), w_{1}(z), w_{2}(z)\right) \rightarrow \varphi(z)+x w_{1}+y w_{2} .
$$

If we are given a functional on the $3 \mathrm{~d}$ phase space (typically kinetic plus potential energy) of the form

$$
F=\iiint_{R_{\epsilon}} f(\phi, \partial \phi, \dot{\phi}, \epsilon) d x d y d z
$$

the induced functional for the rod is obtained by substituting $\phi(x, y, z)=\varphi(z)+x w_{1}(z)+y w_{2}(z)$ in the $3 \mathrm{~d}$ energy, namely

$$
F \circ \mathcal{K}_{\epsilon}=\iiint_{R_{\epsilon}} f\left(\varphi(z)+x w_{1}(z)+y w_{2}(z), \partial\left(\varphi+x w_{1}+y w_{2}\right), \dot{\varphi}+x \dot{w}_{1}+y \dot{w}_{2}\right) d x d y d z .
$$

Suppose that the kinetic energy for the $3 \mathrm{~d}$ elastic body is

$$
\frac{1}{2}\left\langle\left(\phi, \dot{\phi}_{1}\right),\left(\phi, \dot{\phi}_{2}\right)\right\rangle_{3 d}=\frac{1}{2} \iiint_{R_{\epsilon}} \dot{\phi}_{1} \cdot \dot{\phi}_{2} d V
$$

then the induced kinetic energy for the rod is

$$
\frac{\pi \epsilon^{2}}{2} \int_{0}^{l} \dot{\varphi} \cdot \dot{\varphi} d z+\frac{\pi \epsilon^{4}}{4} \int_{0}^{l}\left(\dot{w}_{1} \cdot \dot{w}_{1}+\dot{w}_{2} \cdot \dot{w}_{2}\right) d z .
$$

We use the corresponding Riemannian metric to identify the tangent bundle and cotangent bundle for the rod, and obtain the Poisson bracket

$$
\begin{aligned}
\left\{F_{1}, F_{2}\right\}_{1 d}^{\epsilon}= & \int_{0}^{l} \frac{1}{\pi \epsilon^{2}}\left(\frac{\delta F_{1}}{\delta \varphi} \frac{\delta F_{2}}{\delta \dot{\varphi}}-\frac{\delta F_{2}}{\delta \varphi} \frac{\delta F_{1}}{\delta \dot{\varphi}}\right) d z \\
& +\int_{0}^{l} \frac{2}{\pi \epsilon^{4}}\left(\frac{\delta F_{1}}{\delta w_{1}} \frac{\delta F_{2}}{\delta \dot{w}_{1}}+\frac{\delta F_{1}}{\delta w_{2}} \frac{\delta F_{2}}{\delta \dot{w}_{2}}-\frac{\delta F_{2}}{\delta w_{1}} \frac{\delta F_{1}}{\delta \dot{w}_{1}}-\frac{\delta F_{2}}{\delta w_{2}} \frac{\delta F_{1}}{\delta \dot{w}_{2}}\right) d z .
\end{aligned}
$$

Theorem 5.1 If $F_{1}, F_{2}$ are two functionals of the form (5.1.1), and $F_{1} \circ \mathcal{K}_{\epsilon}, F_{2} \circ \mathcal{K}_{\epsilon}$ their counterparts for the rod, then

$$
\left\{F_{1}, F_{2}\right\}_{3 d}^{\epsilon}=\left\{F_{1} \circ \mathcal{K}_{\epsilon}, F_{2} \circ \mathcal{K}_{\epsilon}\right\}_{1 d}^{\epsilon}+O\left(\epsilon^{3}\right)
$$




\subsection{Constrained and Unconstrained Limiting Models}

As in the previous sections, by studying the dynamics of the limiting model, one obtains either a unconstrained director model or a constrained-director model, depending on different assumptions on the Hamiltonian functional.

For example, consider the Hamiltonian

$$
H=\iiint_{R_{\epsilon}}\left(\frac{1}{2} \dot{\phi} \cdot \dot{\phi}+g(x, y, z, \phi, \partial \phi, \epsilon)\right) d x d y d z
$$

The corresponding limiting equations have the form

$$
\begin{aligned}
\frac{\partial^{2} \varphi}{\partial t^{2}} & =O(1) \\
\frac{\partial^{2} w_{1}}{\partial t^{2}} & =\frac{1}{\epsilon^{2}} \frac{\partial g}{\partial v_{1}}+O(1), \\
\frac{\partial^{2} w_{2}}{\partial t^{2}} & =\frac{1}{\epsilon^{2}} \frac{\partial g}{\partial v_{2}}+O(1),
\end{aligned}
$$

where $v_{1}=\partial \varphi / \partial x$ and $v_{2}=\partial \varphi / \partial y$. If we assume that

$$
\frac{\partial g}{\partial v_{1}}=O\left(\epsilon^{2}\right), \frac{\partial g}{\partial v_{2}}=O\left(\epsilon^{2}\right)
$$

then we are lead to unconstrained 2-director models in the limit $\epsilon \rightarrow 0$.

As with shells, this situation arises if one starts with a specific isotropic $3 \mathrm{~d}$ elasticity model with an energy density of the form

$$
\frac{1}{2} \dot{\phi} \cdot \dot{\phi}+g_{\text {isotropic }}\left(x, y, z, \phi, \frac{\partial \phi}{\partial x}, \frac{\partial \phi}{\partial y}, \frac{\partial \phi}{\partial z}\right) .
$$

Then consider a scaled Hamiltonian

$$
\begin{aligned}
H & =\iiint_{R_{\epsilon}}\left(\frac{1}{2} \dot{\phi} \cdot \dot{\phi}+g\left(x, y, z, \phi, \frac{\partial \phi}{\partial x}, \frac{\partial \phi}{\partial y}, \frac{\partial \phi}{\partial z}, \epsilon\right)\right) d x d y d z \\
& :=\iiint_{R_{\epsilon}}\left(\frac{1}{2} \dot{\phi} \cdot \dot{\phi}+g_{\text {isotropic }}\left(x, y, z, \phi, \epsilon \frac{\partial \phi}{\partial x}, \epsilon \frac{\partial \phi}{\partial y}, \frac{\partial \phi}{\partial z}\right)\right) d x d y d z
\end{aligned}
$$

In particular, if $g$ is quadratic plus higher order terms in $\partial \phi / \partial x$ and $\partial \phi / \partial y$, then it satisfies the hypotheses needed to get an unconstrained limiting model. The Hamiltonian for the limiting model then is given in terms of the variables $\phi(x, y, z)=\varphi(z)+x w_{1}+y w_{2}$ and the scaled variables $\bar{w}_{1}=\epsilon w_{1}$ and $\bar{w}_{2}=\epsilon w_{2}$

$$
H_{\text {rod }}=\int_{0}^{l}\left(\frac{1}{2}\left(\dot{\varphi} \cdot \dot{\varphi}+\dot{\bar{w}}_{1} \cdot \dot{\bar{w}}_{1}+\dot{\bar{w}}_{2} \cdot \dot{\bar{w}}_{2}\right)+g_{\text {rod }}\left(z, \varphi, \bar{w}_{1}, \bar{w}_{2}, \frac{\partial \varphi}{\partial z}, \frac{\partial \bar{w}_{1}}{\partial z}, \frac{\partial \bar{w}_{2}}{\partial z}\right)\right) d z
$$

where

$$
g_{\text {rod }}\left(z, \varphi, \bar{w}_{1}, \bar{w}_{2}, \frac{\partial \varphi}{\partial z}, \frac{\partial \bar{w}_{1}}{\partial z}, \frac{\partial \bar{w}_{2}}{\partial z}\right)=g_{\text {isotropic }}\left(0,0, z, \varphi, \bar{w}_{1}, \bar{w}_{2}, \frac{\partial \varphi}{\partial z}\right)
$$


Then the limiting system has two unconstrained directors.

If, on the other hand, $\partial g / \partial v_{1} \neq O\left(\epsilon^{2}\right), \partial g / \partial v_{2} \neq O\left(\epsilon^{2}\right)$, which has to be evaluated for $\phi=$ $\varphi+x w_{1}+y w_{2}$, then one in general obtains constrained 2-director models in the limit.

Suppose that

$$
\operatorname{det}\left(\frac{\partial^{2} g}{\partial v_{1} \partial v_{2}}\right) \neq 0
$$

Then one can solve for $w_{1}$ and $w_{2}$ in terms of $\varphi$ :

$$
w_{1}=\mathcal{L}_{1}(\varphi), w_{2}=\mathcal{L}_{2}(\varphi)
$$

from the equations

$$
\left.\frac{\partial g}{\partial v_{1}}\right|_{\phi=\varphi(z)+x w_{1}+y w_{2}}=O(\epsilon),\left.\quad \frac{\partial g}{\partial v_{2}}\right|_{\phi=\varphi(z)+x w_{1}+y w_{2}}=O(\epsilon) .
$$

In this case the configuration space is the set

$$
\mathcal{M}_{1 \mathrm{~cd}}=\left\{\varphi: \mathbb{R} \rightarrow \mathbb{R}^{3}\right\}
$$

and the Poisson bracket is given by

$$
\left\{F_{1}, F_{2}\right\}_{1 \mathrm{~cd}}=\int\left(\frac{\delta F_{1}}{\delta \varphi} \frac{\delta F_{2}}{\delta \dot{\varphi}}-\frac{\delta F_{2}}{\delta \varphi} \frac{\delta F_{1}}{\delta \dot{\varphi}}\right) d z
$$

We introduce the map $\mathcal{I}_{\epsilon}: T M_{1 \mathrm{~cd}} \mapsto T M_{3 d}$ as the tangent lift of the map

$$
\varphi(z) \mapsto \varphi(z)+x \mathcal{L}_{1}+y \mathcal{L}_{2}
$$

The map $\mathcal{I}_{\epsilon}$ is an almost-Poisson embedding, in the sense that

$$
\left\{F_{1} \circ \mathcal{I}_{\epsilon}, F_{2} \circ \mathcal{I}_{\epsilon}\right\}_{3 d}^{\epsilon}=\left\{F_{1}, F_{2}\right\}_{1 d}^{\epsilon} \circ \mathcal{I}_{\epsilon}+O\left(\epsilon^{3}\right)
$$

where $F_{1}$ is of the form

$$
\iiint_{R_{\epsilon}}\left(\frac{1}{2} m \dot{\phi} \cdot \dot{\phi}+g\left(x, y, z, \phi, \frac{\partial \phi}{\partial x}, \frac{\partial \phi}{\partial y}, \frac{\partial \phi}{\partial z}, \epsilon\right)\right) d x d y d z
$$

where $m$ is the mass density, $g$ satisfies

$$
\left.\frac{\partial g}{\partial v_{1}}\right|_{\phi=\varphi+x \mathcal{L}_{1}+y \mathcal{L}_{2}}=O\left(\epsilon^{2}\right),\left.\quad \frac{\partial g}{\partial v_{2}}\right|_{\phi=\varphi+x \mathcal{L}_{1}+y \mathcal{L}_{2}}=O\left(\epsilon^{2}\right),
$$

and $F_{2}$ is arbitrary. 


\section{The Kirchhoff Elastica as a Limit of a 3d Saint Venant-Kirchhoff Material}

\subsection{Introduction}

In this section we apply our method to 3d Saint Venant-Kirchhoff materials. As outlined above, the stored energy density of such a material is given by $g(x, y, z, \phi, D \phi)=W\left(D \phi^{T} D \phi\right)$, where

$$
W\left(D \phi^{T} D \phi\right)=\frac{\mu}{4}\left(\sum_{i, j=1}^{3}\left(z_{i} \cdot z_{j}-\delta_{i j}\right)^{2}\right)+\frac{\lambda}{8}\left(\sum_{i=1}^{3}\left(\left\|z_{i}\right\|^{2}-1\right)\right)^{2} .
$$

Here we used $z_{1}:=\partial \phi / \partial x, z_{2}:=\partial \phi / \partial y, z_{3}:=\partial \phi / \partial z$ and Cartesian coordinates $x, y, z$ in $\mathbb{R}^{3}$. Introducing an additional inextensibility constraint will lead to an unconstrained one-director Kirchhoff rod model, the stored energy density of which only depends on curvature and torsion of the rod and the derivative of the director variable along the rod.

In case one starts with a 2d Saint Venant-Kirchhoff material instead of a 3d one, the asymptotic procedure will lead to the well-known Euler elastica as a limit model.

\subsection{A Limiting Director Model for Rods}

To apply our general theory, we make the Ansatz

$$
\phi(x, y, z)=\varphi(z)+x w_{2}(z)+y w_{3}(z)
$$

for a configuration of the $3 \mathrm{~d}$ material and determine the director fields $w_{2}$ and $w_{3}$ from the equations

$$
\begin{aligned}
\frac{\partial g}{\partial v_{1}} & =O(\epsilon), \\
\frac{\partial g}{\partial v_{2}} & =O(\epsilon) .
\end{aligned}
$$

( We changed the numbering of the director fields slightly to make the discussion of inextensible rods in the next section more transparent.) To determine $w_{2}$ and $w_{3}$ so that these equations are satisfied we use the following lemma:

Lemma 6.1 Let $h\left(\varphi_{z}\right)=\left\|\varphi_{z}\right\|^{2}-1$. If

$$
-\frac{\lambda h\left(\varphi_{z}\right)}{2(\mu+\lambda)}>-1
$$

then the equations

$$
\begin{aligned}
& \frac{\partial W}{\partial z_{1}}\left(\bar{w}_{2}, \bar{w}_{3}, z_{3}\right)=O(\epsilon) \\
& \frac{\partial W}{\partial z_{2}}\left(\bar{w}_{2}, \bar{w}_{3}, z_{3}\right)=O(\epsilon)
\end{aligned}
$$


have solutions which satisfy

$$
\begin{aligned}
\bar{w}_{2} \cdot \varphi_{z} & =0 \\
\bar{w}_{3} \cdot \varphi_{z} & =0 \\
\left\|\bar{w}_{2}\right\|^{2}-1 & =-\frac{\lambda h\left(\varphi_{z}\right)}{2(\mu+\lambda)}, \\
\left\|\bar{w}_{3}\right\|^{2}-1 & =-\frac{\lambda h\left(\varphi_{z}\right)}{2(\mu+\lambda)} .
\end{aligned}
$$

Proof We write

$$
\begin{aligned}
W= & \frac{\mu}{4}\left(\sum_{\alpha, \beta=1}^{2}\left(z_{\alpha} \cdot z_{\beta}-\delta_{\alpha \beta}\right)^{2}\right)+\frac{\lambda}{8}\left(\sum_{\alpha=1}^{2}\left(\left\|z_{\alpha}\right\|^{2}-1\right)\right)^{2} \\
& +\frac{\mu}{2}\left(\sum_{\alpha}^{2}\left(z_{\alpha} \cdot z_{3}\right)^{2}\right)+\frac{2 \mu+\lambda}{8}\left(\left\|z_{3}\right\|^{2}-1\right)^{2} \\
& +\frac{\lambda}{4}\left(\left\|z_{3}\right\|^{2}-1\right)\left(\sum_{\alpha}^{2}\left(\left\|z_{\alpha}\right\|^{2}-1\right)\right) .
\end{aligned}
$$

We only consider $\left(z_{1}, z_{2}, z_{3}\right)$ for which $W$ is minimal with respect to $\left(z_{1}, z_{2}\right)$. For $W$ to be minimal, $z_{1}, z_{2}, z_{3}$ have to be orthogonal. Denote $Y_{1}:=\left\|z_{1}\right\|^{2}-1, Y_{2}:=\left\|z_{2}\right\|^{2}-1$ and $\bar{h}:=\left\|z_{3}\right\|^{2}-1$, then

$$
W=\frac{\mu}{4}\left(Y_{1}^{2}+Y_{2}^{2}\right)+\frac{\lambda}{8}\left(Y_{1}+Y_{2}\right)^{2}+\frac{2 \mu+\lambda}{8} \bar{h}^{2}+\frac{\lambda}{4} \bar{h}\left(Y_{1}+Y_{2}\right) .
$$

Thus, $\partial W / \partial Y_{1}=\partial W / \partial Y_{2}=0$ is equivalent to

$$
\begin{aligned}
\frac{\mu}{2} Y_{1}+\frac{\lambda}{4}\left(Y_{1}+Y_{2}\right)+\frac{\lambda}{4} \bar{h} & =0, \\
\frac{\mu}{2} Y_{2}+\frac{\lambda}{4}\left(Y_{1}+Y_{2}\right)+\frac{\lambda}{4} \bar{h} & =0 .
\end{aligned}
$$

In case that $Y_{1} \geq-1$, the solution to these equations is

$$
Y_{1}=Y_{2}=-\frac{\lambda \bar{h}}{2(\mu+\lambda)}
$$

The corresponding potential energy density is

$$
W_{\min }=\left(\frac{2 \mu+\lambda}{8}\right) \bar{h}^{2}-\frac{1}{8(\lambda+\mu)} \lambda^{2} \bar{h}^{2} .
$$

This lemma shows that to solve the equations (6.2.1) and (6.2.2), one has to choose the director fields $w_{2}$ and $w_{3}$ orthogonal to $\varphi_{z}$ and both to be of squared length equal to

$$
1-\frac{\lambda h\left(\varphi_{z}\right)}{2(\mu+\lambda)}
$$


The stored energy density of the limit rod model then is

$$
g_{\text {rod }}=\left(\frac{2 \mu+\lambda}{8}\right) h^{2}-\frac{1}{8(\lambda+\mu)} \lambda^{2} h^{2},
$$

or

$$
\lim _{\epsilon \rightarrow 0} \frac{2}{\epsilon^{2}} V \circ K_{\epsilon}=\left(\frac{2 \mu+\lambda}{8}\right) h\left(\varphi_{z}\right)^{2}-\frac{\lambda^{2}}{8(\lambda+\mu)} h\left(\varphi_{z}\right)^{2}
$$

We see that the limiting procedure applied to a Saint Venant-Kirchhoff material leads to an unconstrained rod model, the energy of which depends only on the first derivatives of the placement field. In the next section we show that one arrives at a limit model of a very different type if one introduces an inextensibility constrain, which is equivalent to setting (6.2.3) to zero (see also the discussion in $\S 4.3)$.

\subsection{An Inextensible Elastica with a Director}

To derive a theory for an inextensible rod, we make the (inextensibility) assumption

$$
\bar{h}(z)=\left\|\partial_{z} \varphi\right\|^{2}-1=0,
$$

which is equivalent to setting the potential energy (6.2.3) to zero. Let $w_{1}:=\partial_{z} \varphi$. From the discussion in the preceding section we see that the director fields $w_{2}, w_{3}$ of the rod should satisfy the following equations:

$$
\begin{aligned}
w_{2} \cdot w_{3} & =0, \\
w_{2} \cdot \varphi_{z} & =0, \\
w_{3} \cdot \varphi_{z} & =0 \\
\left\|w_{2}\right\|^{2}-1 & =0 \\
\left\|w_{3}\right\|^{2}-1 & =0 .
\end{aligned}
$$

If we assume that

$$
\operatorname{det}\left(w_{1}, w_{2}, w_{3}\right)>0,
$$

then the director fields $w_{2}, w_{3}$ are determined by these constraints up to a rotation about $w_{1}$. In particular, we have $w_{3}=\varphi_{z} \times w_{2}$. After a calculation one finds (neglecting higher order terms in $\epsilon)$

$$
V=\frac{2 \mu+\lambda}{8} \pi \epsilon^{4} \int\left(\left\langle\varphi_{z},\left(w_{2}\right)_{z}\right\rangle^{2}+\left\langle\varphi_{z},\left(w_{3}\right)_{z}\right\rangle^{2}\right) d z+\frac{\mu}{8} \pi \epsilon^{4} \int\left\langle w_{2}, w_{1} \times\left(w_{2}\right)_{z}\right\rangle^{2} d z
$$

Because $\left\langle\varphi_{z z}, \varphi_{z}\right\rangle=0$ and $w_{2}$ and $w_{3}$ are orthonormal, one has

$$
\varphi_{z z}=\left\langle\varphi_{z z}, w_{2}\right\rangle w_{2}+\left\langle\varphi_{z z}, w_{3}\right\rangle w_{3}
$$

Using the equations

$$
\left\langle\varphi_{z},\left(w_{i}\right)_{z}\right\rangle=\partial_{z}\left\langle\varphi_{z}, w_{i}\right\rangle-\left\langle\varphi_{z z}, w_{i}\right\rangle \quad(i=2,3)
$$


and the fact that $\varphi_{z}, w_{2}, w_{3}$ are orthonormal in equation (6.3.1) we get

$$
\varphi_{z z}=-\left\langle\varphi_{z},\left(w_{2}\right)_{z}\right\rangle w_{2}-\left\langle\varphi_{z},\left(w_{3}\right)_{z}\right\rangle w_{3}
$$

and

$$
\left\|\varphi_{z z}\right\|^{2}=\left\langle\varphi_{z},\left(w_{2}\right)_{z}\right\rangle^{2}+\left\langle\varphi_{z},\left(w_{3}\right)_{z}\right\rangle^{2} .
$$

Writing $a:=\left\|\varphi_{z z}\right\|$ for the curvature function of the curve $z \mapsto \varphi(z)$ and neglecting higher order terms in $\epsilon$ then yields

$$
V=\frac{2 \mu+\lambda}{8} \pi \epsilon^{4} \int a^{2} d z+\frac{\mu}{8} \pi \epsilon^{4} \int\left\langle w_{2}, w_{1} \times\left(w_{2}\right)_{z}\right\rangle^{2} d z .
$$

If $a \neq 0$, we may choose the director field $w_{2}$ pointwise collinear to the field $\varphi_{z z}$ :

We introduce the Frenet frame $v_{1}, v_{2}, v_{3}$, where $v_{1}(z)=\partial_{z} \varphi(z), v_{2}(z)$ is a unit vector in the direction of the curvature vector of $\varphi$ at $\varphi(z)$, and $v_{3}(z)=v_{1}(z) \times v_{2}(z)$ denotes the binormal vector of $\varphi$ at $\varphi(z)$. The Frenet equations

$$
\begin{aligned}
& \frac{d v_{1}}{d z}=a v_{2}, \\
& \frac{d v_{2}}{d z}=-a v_{1}-c v_{3}, \\
& \frac{d v_{3}}{d z}=c v_{2}
\end{aligned}
$$

hold, where $a(z)$ is the curvature of $\varphi$ at $\varphi(z)$ and $c(z)$ is the torsion (see, for example, do Carmo [1976]).

We now can write

$$
\begin{aligned}
& w_{1}=v_{1}, \\
& w_{2}=A v_{2}, \\
& w_{3}=A v_{3},
\end{aligned}
$$

where $A(z) \in S O(2)$ describes a rotation at $w_{1}(z)$ by an angle $\Theta(z)$. We plug the Ansatz

$$
\phi(x, y, z)=\varphi(z)+x w_{2}(z)+y w_{3}(z)
$$

into the (kinetic+potential) energy density of the 3d Saint Venant-Kirchhoff material and integrate over the reference configuration to get the energy $H$ for the inextensible rod. After a calculation, one finds that

$$
H=K+V
$$

where

$$
\begin{aligned}
& K= \int\left[\frac{1}{2} \pi \epsilon^{2} \dot{\varphi}^{2}+\frac{\pi}{4} \epsilon^{4} \dot{\Theta}^{2}+\frac{\pi}{4} \epsilon^{4}\left\{\left\langle v_{1}, v_{2} \times \dot{v}_{2}\right\rangle+\left\langle v_{1}, v_{3} \times \dot{v}_{3}\right\rangle\right\} \dot{\Theta}+\frac{\pi}{8} \epsilon^{4}\left\{\left\|\dot{v}_{2}\right\|^{2}+\left\|\dot{v}_{3}\right\|^{2}\right\}\right] d z \\
&=\int\left[\frac{1}{2} \pi \epsilon^{2} \dot{\varphi}^{2}+\frac{\pi}{4} \epsilon^{4} \dot{\Theta}^{2}+\frac{\pi}{2 a^{2}} \epsilon^{4}\left\langle\varphi_{z} \times \varphi_{z z}, \dot{\varphi}_{z z}\right\rangle \dot{\Theta}+\frac{\pi}{4 a^{2}} \epsilon^{4}\left\|\dot{\varphi}_{z z}\right\|^{2}-\frac{\pi}{4 a^{4}} \epsilon^{4}\left\langle\varphi_{z z}, \dot{\varphi}_{z z}\right\rangle^{2}\right. \\
&\left.+\frac{\pi}{8 a^{2}} \epsilon^{4}\left\|\varphi_{z z} \times \dot{\varphi}_{z}\right\|^{2}+\frac{\pi}{4 a^{2}} \epsilon^{4}\left\langle\dot{\varphi}_{z} \times \varphi_{z z}, \varphi_{z} \times \dot{\varphi}_{z z}\right\rangle\right] d z,
\end{aligned}
$$


and (neglecting terms of higher order in $\epsilon$ )

$$
V=\int\left[\frac{\pi \epsilon^{4}}{8} \mu\left(c+\Theta_{z}\right)^{2}+\frac{\pi \epsilon^{4}}{8}(2 \mu+\lambda) a^{2}\right] d z .
$$

In Landau and Lifshitz [1959] the quantity $\Theta_{z}$ is called the torsion angle, in case the rod is unbent.

The function $H$ may be interpreted in different ways. On the one hand, one can think of it as the energy function for a two-director rod model, where the directors are constrained to be orthonormal and to be orthogonal to the tangent vector of the centerline of the rod. On the other hand one might interpret $H$ as the energy function of an unconstrained one-director rod model with variables $\varphi$ (position of centerline of the rod) and $\Theta$ (twist of the normal cross-sections to the centerline of the rod). Here $\Theta$ is interpreted as the director variable.

One might also think of $H$ as the energy function of a three-director rod model, following Maddocks [1984], who introduces two directors $d_{1}, d_{2}:[0,1] \rightarrow \mathbb{R}^{3}$ that are orthonormal and orthogonal to the tangent vector of the axis $r:[0,1] \rightarrow \mathbb{R}^{3}$ of the rod. He imposes the additional constraint $\left\|r^{\prime}\right\|=1$, puts $d_{1}:=r^{\prime}$, and regards the resulting rod model as a three-director rod model with directors $d_{1}, d_{2}, d_{3}$.

To understand how the energy function behaves under rescaling, let

$$
\begin{aligned}
\epsilon^{\alpha} \bar{z} & :=z, \\
\epsilon^{\beta} \bar{\varphi} & :=\varphi, \\
\epsilon^{\gamma} \bar{\theta} & :=\theta, \\
\epsilon^{\delta} \bar{t} & :=t, \\
\epsilon^{\rho} \bar{\mu} & :=\mu, \\
\epsilon^{\sigma} \bar{\lambda} & :=\lambda .
\end{aligned}
$$

After rescaling, the potential energy is given by

$$
\begin{aligned}
\bar{V}= & \int\left[\frac{\pi}{4} \epsilon^{4-\alpha+2 \gamma+\rho} \bar{\mu}\left(\bar{\Theta}_{\bar{z}}\right)^{2}-\frac{\pi}{2} \epsilon^{4-\beta+\gamma+\rho} \bar{\mu} \bar{c} \bar{\Theta}_{\bar{z}}\right. \\
& \left.+\frac{\pi}{4} \epsilon^{4+\alpha-2 \beta+\rho} \bar{\mu} \bar{c}^{2}+\frac{\pi}{2} \epsilon^{4+\alpha-2 \beta+\rho} \bar{\mu} \bar{a}^{2}+\frac{\pi}{4} \epsilon^{4+\alpha-2 \beta+\sigma} \bar{\lambda} \bar{a}^{2}\right] d \bar{z}
\end{aligned}
$$

where $\bar{a}$ denotes the curvature of the curve $\bar{\varphi}$ and $\bar{c}$ its torsion. The rescaled kinetic energy is given by

$$
\begin{aligned}
\bar{K}= & \int\left[\frac{1}{2} \epsilon^{2+\alpha+2 \beta-2 \delta} \dot{\bar{\varphi}}^{2}+\frac{\pi}{4} \epsilon^{4+\alpha+2 \gamma-2 \delta} \dot{\bar{\Theta}}^{2}+\frac{\pi}{2 \bar{a}^{2}} \epsilon^{4-4 \alpha+5 \beta+\gamma-2 \delta}\left\langle\bar{\varphi}_{\bar{z}} \times \bar{\varphi}_{\bar{z} \bar{z}}, \dot{\bar{\varphi}}_{\bar{z} \bar{z}}\right\rangle \dot{\bar{\Theta}}\right. \\
& +\frac{\pi}{4 \bar{a}^{2}} \epsilon^{4-3 \alpha+4 \beta-2 \delta}\left\|\dot{\bar{\varphi}}_{\bar{z} \bar{z}}\right\|^{2}-\frac{\pi}{4 \bar{a}^{4}} \epsilon^{4-7 \alpha+6 \beta-2 \delta}\left\langle\bar{\varphi}_{\bar{z} \bar{z}}, \dot{\bar{\varphi}}_{\bar{z} \bar{z}}\right\rangle^{2}+\frac{\pi}{8 \bar{a}^{2}} \epsilon^{4-5 \alpha+6 \beta-2 \delta}\left\|\bar{\varphi}_{\bar{z} \bar{z}} \times \dot{\bar{\varphi}}_{\bar{z}}\right\|^{2} \\
& \left.+\frac{\pi}{4 \bar{a}^{2}} \epsilon^{4-5 \alpha+6 \beta-2 \delta}\left\langle\dot{\bar{\varphi}}_{\bar{z}} \times \bar{\varphi}_{\bar{z} \bar{z}}, \bar{\varphi}_{\bar{z}} \times \dot{\bar{\varphi}}_{\bar{z} \bar{z}}\right\rangle\right] d \bar{z} .
\end{aligned}
$$

Here, $\dot{\bar{\varphi}}$ and $\dot{\bar{\Theta}}$ are the derivatives of $\bar{\varphi}$ and $\bar{\Theta}$ with respect to the rescaled time-variable $\bar{t}$. If one 
chooses $\beta$ arbitrarily and sets

$$
\begin{aligned}
\alpha & :=2 \beta-1, \\
\gamma & :=\beta-1, \\
\delta & :=\frac{1}{2}(4 \beta+1), \\
\rho & :=-3, \\
\sigma & :=-3,
\end{aligned}
$$

then the equations for potential and kinetic energy become

$$
\bar{V}=\int\left[\frac{\pi}{4} \bar{\mu} \bar{\Theta}_{\bar{z}}^{2}-\frac{\pi}{2} \bar{\mu} \bar{c} \bar{\Theta}_{\bar{z}}+\frac{\pi}{4} \bar{\mu} \bar{c}^{2}+\frac{\pi}{2} \bar{\mu} \bar{a}^{2}+\frac{\pi}{4} \bar{\lambda} \bar{a}^{2}\right] d \bar{z}
$$

and

$$
\begin{aligned}
\bar{K}= & \int\left[\frac{1}{2} \dot{\bar{\varphi}}^{2}+\frac{\pi}{4} \dot{\bar{\Theta}}^{2}+\frac{\pi}{2 \bar{a}^{2}} \epsilon^{6-6 \beta}\left\langle\bar{\varphi}_{\bar{z}} \times \bar{\varphi}_{\bar{z} \bar{z}}, \dot{\bar{\varphi}}_{\bar{z} \bar{z}}\right\rangle \dot{\bar{\Theta}}+\frac{\pi}{4 a^{2}} \epsilon^{6-6 \beta}\left\|\dot{\bar{\varphi}}_{\bar{z} \bar{z}}\right\|^{2}\right. \\
& \left.-\frac{\pi}{4 \bar{a}^{4}} \epsilon^{10-12 \beta}\left\langle\bar{\varphi}_{\bar{z} \bar{z}}, \dot{\bar{\varphi}}_{\bar{z} \bar{z}}\right\rangle^{2}+\frac{\pi}{8 \bar{a}^{2}} \epsilon^{8-8 \beta}\left\|\bar{\varphi}_{\bar{z} \bar{z}} \times \dot{\bar{\varphi}}_{\bar{z}}\right\|^{2}+\frac{\pi}{4 \bar{a}^{2}} \epsilon^{8-8 \beta}\left\langle\dot{\bar{\varphi}}_{\bar{z}} \times \bar{\varphi}_{\bar{z} \bar{z}}, \bar{\varphi}_{\bar{z}} \times \dot{\bar{\varphi}}_{\bar{z} \bar{z}}\right\rangle\right] d \bar{z}
\end{aligned}
$$

In particular, if we choose $\beta:=1 / 2$, then $\alpha=0$ and the kinetic energy has the form

$$
\begin{aligned}
\bar{K}= & \int\left[\frac{1}{2} \dot{\bar{\varphi}}^{2}+\frac{\pi}{4} \dot{\bar{\Theta}}^{2}+\frac{\pi}{2 \bar{a}^{2}} \epsilon^{3}\left\langle\bar{\varphi}_{\bar{z}} \times \bar{\varphi}_{\bar{z} \bar{z}}, \dot{\bar{\varphi}}_{\bar{z} \bar{z}}\right\rangle \dot{\bar{\Theta}}+\frac{\pi}{4 a^{2}} \epsilon^{3}\left\|\dot{\bar{\varphi}}_{\bar{z} \bar{z}}\right\|^{2}\right. \\
& \left.-\frac{1 \pi}{4 \bar{a}^{4}} \epsilon^{4}\left\langle\bar{\varphi}_{\bar{z} \bar{z}}, \dot{\bar{\varphi}}_{\bar{z} \bar{z}}\right\rangle^{2}+\frac{\pi}{8 \bar{a}^{2}} \epsilon^{4}\left\|\bar{\varphi}_{\bar{z} \bar{z}} \times \dot{\bar{\varphi}}_{\bar{z}}\right\|^{2}+\frac{\pi}{4 \bar{a}^{2}} \epsilon^{4}\left\langle\dot{\bar{\varphi}}_{\bar{z}} \times \bar{\varphi}_{\bar{z} \bar{z}}, \bar{\varphi}_{\bar{z}} \times \dot{\bar{\varphi}}_{\bar{z} \bar{z}}\right\rangle\right] d \bar{z} .
\end{aligned}
$$

Thus, after neglecting terms of higher order in $\epsilon$, the kinetic energy is given by

$$
\bar{K}=\int\left[\frac{1}{2} \dot{\bar{\varphi}}^{2}+\frac{\pi}{4} \dot{\bar{\Theta}}^{2}\right] d \bar{z} .
$$

Maddocks [1984] discusses the equilibrium problem for a three-director rod model in Euclidean three space and Caflisch and Maddocks [1984] discuss the dynamics of a planar rod model. Our model is a dynamic three dimensional one-director model and we provide a justification for the expression of the kinetic energy. The justification for such expressions seems to be a general problem in the direct approach to the theory of Cosserat continua (compare Antman [1995], p. 262), whereas we derive our energy functional by an asymptotic procedure from the $3 \mathrm{~d}$ theory.

\subsection{The Euler-Kirchhoff Elastica}

Introducing the additional holonomic constraint $\Theta=0$ in the one-director model just derived yields a Kirchhoff elastica model whose stored energy density depends only on the curvature and torsion of the rod, namely

$$
\bar{V}=\int\left[\frac{\pi}{8} \bar{\mu} \bar{c}^{2}+\frac{\pi}{8}(2 \bar{\mu}+\bar{\lambda}) \bar{a}^{2}\right] d \bar{z}
$$


Note that if one starts from a $2 \mathrm{~d}$ Saint-Venant Kirchhoff material, one arrives at the classical planar Euler elastica as a limit model without having to impose any extra constraint. The stored energy density of this rod model is (modulo a constant factor) given by the square of the curvature:

$$
V_{E}=\int a^{2} d z
$$

(see Love [1944]).

One can also combine the constraint $\Theta=0$ and the assumption that the rod is planar in the following way. We say a rod is planar if it is invariant under a one-parameter group of translations in $\mathbb{R}^{3}$, in which case $c=0$. Now fix a one-parameter group of translations in $\mathbb{R}^{3}$. The set

$$
\{(\varphi, \dot{\varphi}, \Theta, \dot{\Theta}), \varphi, \dot{\varphi} \text { are planar ( with respect to the group of translations ), } \Theta=\dot{\Theta}=0\}
$$

is an invariant submanifold of the Hamiltonian system of the one-director rod model derived in the last subsection. This can be seen as follows. The Hamiltonian function is invariant under translations in $\varphi$, so if at $t=0, \varphi, \dot{\varphi}$ are planar (with respect to the one-parameter group of translations), they will remain so for $t>0$ and hence $c=0$. Then the Hamiltonian function splits as the sum of two functions, the first only involves $\Theta, \dot{\Theta}$, and the second only involves $\varphi, \dot{\varphi}$. Moreover, $\Theta=0$ is a solution of the first Hamiltonian system.

The elastica has received much attention in recent years from the viewpoint of infinite-dimensional Hamiltonian systems as well as from the viewpoint of finite-dimensional Hamiltonian systems. For example, the elastica occurs as a 'soliton' solution of the vortex filament equation, which is an infinite-dimensional Hamiltonian system closely related to the nonlinear Schrödinger equation (see Langer and Perline [1991]). Here, "soliton solution" means that the elastica moves like a heavy rigid body under the dynamics of the vortex filament equations. The link between the equations for a top and the equations for the elastica can be found in Love [1944] and this link can be used to integrate the equations; the link has also been exploited in, for example, Mielke and Holmes [1988].

In Foltinek [1994], the integrability of the elastica equation is discussed from the viewpoint of finite dimensional Hamiltonian systems with symmetries. In this approach, as in Love [1944] and Mielke and Holmes [1988], the arc length parameter of the rod is interpreted as a time-like variable. Integrals of motion can then be derived using the theory of reduction of Hamiltonian systems with symmetry and methods of (singular) reduction is used to analyze the problem.

An instance for the ubiquity of the elastica can be found in Abresch [1987] who shows that planar $\lambda_{1}$-curvature lines (where $\lambda_{1}<\lambda_{2}$ denote the principal curvatures) on a torus with constant mean curvature are solutions of the elastica equations. (As in the work of Langer and Perline, there is a connection to vortex theory: Constant mean curvature tori can be classified with the help of the sinh-Gordon equation, which also describes vortices in Plasma Physics.)

\section{Acknowledgments}

The authors wish to especially thank the late Juan-Carlos Simo who inspired and helped initiate this work. We also thank John Maddocks and Annie Raoult for useful comments. Zhong Ge and Hans-Peter Kruse thank Jerry Marsden and the University of California for their hospitality during their visits.

The authors wish to acknowledge the following research support: the Ministry of Colleges and Universities of Ontario and the Natural Sciences and Engineering Research Council of Canada (ZG), 
partial support by the Humboldt Foundation during a stay at the Department of Mathematics, University of California, Berkeley, CA 94720 and by the DFG under contract Sch 233/3-1 (HPK) and partial support by the Fields Institute (JEM).

\section{References}

Abresch, U. [1987] Constant mean curvature tori in terms of elliptic functions. J. Reine u. Angew. Math. 374, 169-192.

Antman, S.S. and W. H. Warner [1967] Dynamical theory of hyperelastic rods. Arch. Rat. Mech. Anal. 23, 135-162.

Antman, S.S. [1972], The theory of rods, Handbuch der Physik Band VIa/2, S. Flügge and C. Truesdell eds., Springer-Verlag, Berlin, 641-703.

Antman, S.S. [1995], Nonlinear Problems of Elasticity, Applied Mathematical Sciences, 107, Springer-Verlag, New York.

Caflisch, R. and J.H. Maddocks [1984] Nonlinear dynamical theory of the elastica. Proc. R. Soc. Edin. 99A, 1-23.

Camassa, R. and D. Holm [1993] An integrable shallow water equation with peaked solitons, Phys. Rev. Lett., 71, 1661-1664.

Ciarlet, P.G. [1980], A justification of the von Kármán equations. Arch. Rat. Mech. Anal. 73, 349-389.

Ciarlet, P.G. [1994] Mathematical shell theory: recent developments and open problems, in $D u$ ration and Change: Fifty years at Oberwolfach, M. Artin, H. Kraft, R. Remmert, Eds., Springer-Verlag, 159-176.

Ciarlet, P.G. and B. Miara [1992], Two dimensional shallow shell equations. Comm. Pure Appl. Math. XLV, 327-360.

Ciarlet, P.G. and V. Lods [1994] Analyse asymptotique des coques linéairement élastiques. III. Une justification du modèle de W. T. Koiter. C. R. Acad. Sci. Paris 319 299-304.

Ciarlet, P.G., V. Lods and B. Miara [1994] Analyse asymptotique des coques linéairement élastiques. II. Coques "en flexion". C. R. Acad. Sci. Paris 319, 95-100, 1994.

Destuynder, P. [1985], A classification of thin shell theories. Acta. Appl. Math. 4,15-63.

do Carmo, M. [1976], Differential Geometry of Curves and Surfaces, Prentice-Hall, Englewood Cliffs, N.J.

Foltinek, K. [1994] The Hamilton theory of elastica. Am. J. Math. 116, 1479-1488.

Fox, D., A. Raoult, and J.C. Simo [1992] Modèles asymptotiques invariants pour des structures minces élastiques. C.R. Acad. Sci. Paris 315, 235-240. 
Fox, D., A. Raoult, and J.C. Simo [1993] A justification of nonlinear properly invariant plate theories. Arch. Rational Mech. Anal. 124, 157-199.

Ge, Z. [1991] Equivariant symplectic difference schemes and generating functions, Physica D 49, $376-386$.

Ge, Z. and J.E. Marsden [1988] Lie-Poisson integrators and Lie-Poisson Hamilton-Jacobi theory, Phys. Lett. A 133, 134-139.

Ge, Z., H.P. Kruse, J.E. Marsden and C. Scovel [1995] Poisson Brackets in the Shallow Water Approximation. Can. Appl. Math. Quart. 3, 277-302.

Ge, Z. and C. Scovel [1994] A Hamiltonian truncation of the shallow water equation. Lett. in Math. Phys. 31, 1-13.

John, F. [1971], Refined interior equations for the elastic shells. Comm. Pure. Appl. Math. 24, 584-675.

Kato, T. [1985] Abstract Differential Equations and Nonlinear Mixed Problems. Lezioni Fermiane, Scuola Normale Superiore, Accademia Nazionale dei Lincei.

Koiter, W.T. [1970], On the foundation of the linear theory of thin elastic shells. Proc. Kon. Nederl. Akad. Wetensch. B69, 1-54.

Landau, L.D. and E.M. Lifshitz [1959], Theory of Elasticity, Addison-Wesley, Reading, Mass.

Langer, J. and R. Perline [1991] Poisson geometry of the filament equation. J. of Nonlinear Sci. 1, 71-94.

Le Dret, H. and A. Raoult [1995] The nonlinear membrane model as a variational limit of nonlinear three-dimensional elasticity. J. Math. Pures Appl. 74, 549-578.

Love, A.E.H [1944] A Treatise on the Mathematical Theory of Elasticity. Dover, New York.

Maddocks, J. [1984] Stability of nonlinearly elastic rods. Arch. Rat. Mech. An. 85, 311-354.

Maddocks, J. [1991] On the stability of relative equilibria. IMA J. Appl. Math. 46, 71-99.

Marsden, J.E. and T.J.R. Hughes [1994] Mathematical Foundations of Elasticity. Dover Publications, N.Y.; reprint of 1983 Prentice Hall edition.

Marsden, J.E., T.S. Ratiu, and G. Raugel [1995] Equations d'Euler dans une coque sphérique mince (The Euler equations in a thin spherical shell preprint.

Mielke, A. and P. Holmes [1988] Spatially complex equilibria of buckled rods. Arch. Rat. Mech. An., 101, 319-348.

Naghdi, P. [1972], The theory of shells and plates. Handbuch der Physik Band VIa/2, S. Flügge and C. Truesdell eds., Springer-Verlag, Berlin, 425-640. 
Simo, J.C., M.S. Rifai, and D.D Fox [1992], On a stress resultant geometrically exact shell models. Part VI: Conserving algorithms for nonlinear dynamics. Comp. Meth. Appl. Mech. Eng. 34, 117-164.

Simo, J.C., J.E. Marsden, and P.S. Krishnaprasad [1988] The Hamiltonian structure of nonlinear elasticity: The material, spatial, and convective representations of solids, rods, and plates, Arch. Rat. Mech. Anal. 104, 125-183.

Simo, J.C., T.A. Posbergh, and J.E. Marsden [1990] Stability of coupled rigid body and geometrically exact rods: block diagonalization and the energy-momentum method, Physics Reports 193, $280-360$.

Simo, J.C., T.A. Posbergh, and J.E. Marsden [1991] Stability of relative equilibria II: Three dimensional elasticity, Arch. Rat. Mech. Anal. 115, 61-100. 